Sahinyan et al. 2021 manuscript

\title{
Application of ATAC-Seq for genome-wide analysis of the chromatin state at single myofiber resolution
}

\section{Korin Sahinyan $^{1,2}$ *, Darren M. Blackburn ${ }^{1,2^{*}}$, Marie-Michelle Simon ${ }^{1,3^{*}}$, Felicia Lazure ${ }^{1,2}$,}

${ }^{1}$ Department of Human Genetics, McGill University, 3640 rue University, Montréal, QC, H3A OC7,

7 Canada.

$8 \quad{ }^{2}$ Lady Davis Institute for Medical Research, Jewish General Hospital, 3755 Chemin de la Côte-Sainte-

9 Catherine, Montréal QC, H3T 1E2, Canada.

$10{ }^{3}$ McGill University and Genome Québec Innovation Centre, Montréal, QC, Canada.

$11{ }^{4}$ Canadian Centre for Computational Genomics, Montréal, QC, Canada; Institute for the Advanced

12 Study of Human Biology, Kyoto University, Kyoto, Japan

$13 \quad{ }^{5}$ Corresponding author: Tel: +15143408222 ext. 26136 Fax: +15143407502

14 vahab.soleimani@mcgill.ca

$15 *$ These authors have contributed equally. 
Sahinyan et al. 2021 manuscript

\section{ABSTRACT}

29 Skeletal myofibers are the main components of skeletal muscle which is the largest tissue in the body.

30 Myofibers are highly adaptive in nature and they can vary in different biological and disease conditions.

31 Therefore, transcriptional and epigenetic studies on myofibers are crucial to discover how chromatin

32 alterations occur in the skeletal muscle under different conditions. However, due to the heterogenous

33 nature of skeletal muscle, studying myofibers in isolation proves to be a challenging task. Single cell

34 sequencing has permitted for the study of the epigenome of isolated myonuclei. While this provides

35 sequencing with high dimensionality, the sequencing depth is lacking, which makes comparisons between

36 different biological conditions difficult. Here we report the first implementation of single myofiber

37 ATAC-Seq, which permits for the sequencing of an individual myofiber at a depth sufficient for peak

38 calling and for comparative analysis of chromatin accessibility under various physiological, physical and

39 disease conditions. Application of this technique revealed significant differences in chromatin

40 accessibility between resting and regenerating myofibers. This technique can lead to wide application in

41 identifying chromatin regulatory elements and epigenetic mechanisms in muscle fibers during

42 development and in muscle-wasting diseases.

\section{INTRODUCTION}

44 Skeletal muscle evolved for contraction and production of force. The main component of skeletal muscle 45 are myofibers which are formed from the fusion of myogenic precursor cells (1) and myofibers are large 46 postmitotic syncytia that are composed of repeating contractile units, called sarcomeres (2). Myofibers 47 exhibit wide variations in their metabolic activity and contractile properties (3). In addition, they have a 48 highly adaptive nature where their size, and the overall skeletal muscle mass, are regulated by complex 49 processes involving rates of protein turnover (4,5) transcriptional (6) and posttranscriptional (7) control of 50 gene expression. Due to their adaptive nature, myofibers can change in response to exercise $(3,8,9)$, aging 51 (10) and diseases, such as sarcopenia $(11,12)$ and cachexia (13). Therefore, the study of the myofiber 52 transcriptome/epigenome can provide key insights into how skeletal muscle adapts and changes under 53 different conditions, and it can potentially lead to discovery of novel therapeutic venues for muscle 54 related diseases.

55 Myofibers also act as a key signaling component of muscle stem cells (MuSCs) (14), which are in turn

56 required for the regeneration of nascent muscle fiber after injury. Skeletal muscle is a very heterogenous

57 tissue composed not only of myofibers and their associated MuSCs, but also numerous different non-

58 myogenic cell types (15). Previous studies using whole muscle sequencing captures not only the

59 myofibers but also the other resident cell types in the muscle, making it challenging to attribute changes 
Sahinyan et al. 2021 manuscript

in transcriptome and epigenome specifically to myofibers as they could be due to changes in other cell types. Recent advances in Next Generation Sequencing (NGS) now allows for high dimensional analysis at a single cell level. Recent studies using these technologies to study muscle tissue, such as single nucleus RNA-Seq and ATAC-Seq have analyzed the transcriptome and epigenome of the myonuclei within the muscle fiber (16-18). However, they present certain limitations where they sequence all myonuclei present in the muscle and cannot distinguish between different myofibers as well as having low sequencing depth with limited capacity of analysis.

Chromatin states play key roles in transcriptional regulation and determination of cellular identity (19). Although the accessible regions make up only $3 \%$ of the total genome, it represents over $90 \%$ of known transcription factor binding sites (20). Differential chromatin accessibility is a determinant of transcriptional gene regulation of different cell types and changes in chromatin accessibility have been shown in different biological and disease conditions such as during development $(21,22)$, cancers $(23,24)$ and neurological disorders $(25,26)$ among many others. Thus, in recent years, the study of epigenetics and

73 chromatin accessibility has become a promising field for the development of novel therapeutics. Today,

74 ATAC-Seq is the widely used method that allows for the successful mapping of the accessible chromatin

75 regions in the genome. ATAC-Seq relies on the hyperactive Tn5 transposase that fragments the accessible

76 regions in the genome while simultaneously ligating sequencing compatible adaptors $(27,28)$. Over the

77 years, ATAC-Seq has been applied to many different cell types and tissues (29-31). However, to our

78 knowledge, it has not been performed on a single myofiber, possibly due to the rigidity of their

79 membrane, high levels of mitochondria (32-34) and the low number of myonuclei that is present in a

80 single myofiber $(35,36)$.

81 Here, we have adapted OMNI-ATAC-seq to determine genome-wide chromatin accessibility of 82 myonuclei contained within a single Extensor Digitorum Longus (EDL) muscle fiber in mouse. The

83 Single myofiber ATAC-Seq (smfATAC-Seq) method that we applied in this study allows for the 84 investigation of the accessible chromatin state of a single myofiber, without the presence of other 85 confounding cell types, with high sequencing depth. Through this method we provide comparative 86 analysis of chromatin accessibility between resting and regenerating myofibers as well as their MuSC

87 progenitors. This method can be used in the future to profile the epigenetic state of myofibers in diseases and under various physiological and physical stimuli and to identify active cis-regulatory elements in muscle fibers. 
Sahinyan et al. 2021 manuscript

\section{RESULTS}

\section{Generation of ATAC-Seq libraries from a single myofiber}

93 A single EDL myofiber of a mouse contains an average of 200-300 myonuclei $(35,36)$, making genome-

94 wide analyses of chromatin state difficult. With the advancements in next generation sequencing (NGS)

95 and the development of the OMNI ATAC-Seq protocol (27), analysis of chromatin accessibility of

96 samples with an input of as low as 500 cells is now possible. However, myofibers present additional

97 challenges with their rigid membrane and high levels of mitochondria (32-34). Here we report a robust

98 protocol for the successful application of ATAC-Seq on a single myofiber from the EDL muscle. Our

99 method relies on the lysis and permeabilization of a single myofiber followed by transposition with a

100 hyperactive Tn5 transposase (27) (Figure 1). DNA fragment sizes obtained from the smfATAC-Seq were

101 of a similar range in size as those obtained from conventional OMNI ATAC-Seq that we have performed

102 on MuSCs that were freshly sorted by Fluorescence Activated Cell Sorting (FACS) (Supplemental Figure

103 1). Furthermore, post sequencing analysis showed that only $0.9-2.09 \%$ of reads were derived from the

104 mitochondria in smfATAC-Seq (Table 1), suggesting that this method is highly efficient for the removal

105 of mitochondria from mitochondria-rich myofibers.

106 smfATAC-Seq can be used to study chromatin accessibility of myofibers under different physiological 107 conditions

108 In addition to adapting the ATAC-Seq method to study the chromatin accessibility of a single myofiber,

109 we also demonstrate the application of this technique for comparative analysis of chromatin accessibility

110 between myofibers under different conditions. For that purpose, we performed ATAC-Seq on myofibers

111 that were in a resting (uninjured) or regenerating (injured) state. Uninjured and injured (7 days post

112 cardiotoxin (CTX) induced injury) myofibers were isolated from wild type (WT) C57Bl/6 mice and

113 smfATAC-Seq was performed to compare the changes in chromatin accessibility during regeneration. In

114 addition, we compared the chromatin accessibility between myonuclei within a single myofiber and

115 freshly isolated MuSCs. This analysis not only identified accessible regions of chromatin in myofibers

116 and MuSCs but it also revealed a repertoire of active cis-regulatory elements in each sample.

117 Apart from the myofibers and their associated MuSCs, skeletal muscle also contains many non-myogenic 118 cells such as endothelial cells, adipocytes, hematopoietic cells, fibroblasts, fibro/adipogenic progenitors 119 (FAPs) and macrophages $(15,37,38)$. Our smfATAC-Seq method allows for the analysis of chromatin 120 accessibility of a single myofiber without the confounding effect of these contaminating cell types. To 121 illustrate the absence of the aforementioned cell types in the ATAC-Seq samples, peaks at the promoter 
Sahinyan et al. 2021 manuscript

122 regions of marker genes of muscle resident cells were searched for. Specifically, Platelet and Endothelial

123 Cell Adhesion Molecule 1 (Pecam1) was used to determine whether endothelial cells were present (39).

124 Similarly, Resistin (Retn) and Cd45 were used as markers for adipocytes and hematopoietic cells,

125 respectively $(40,41)$. The cell Surface Antigen Thyl was the marker selected for fibroblasts (42). In

126 addition, Lymphocyte antigen 6a (Ly6a) and Adhesion G protein-coupled receptor E1 (ADGRE1) were

127 selected for fibro/adipogenic progenitors (FAPs) and macrophages, respectively $(43,44)$. None of these

128 marker genes had ATAC-Seq peaks at their promoters, indicating that only a single myofiber is processed

129 without any other contaminating cell types. (Supplemental Figure 2).

130 smfATAC-Seq may be applied to myofibers under different conditions. For instance, we applied smf-

131 ATAC-Seq to analyze the chromatin accessibility of myofibers under resting and CTX-mediated injury

132 conditions. In a disease condition or in injury, not all myofibers undergo damage or regenerate

133 simultaneously. Therefore, individual myofibers within a muscle can be in different physiological and

134 disease condition asynchronously (45). Damaged or regenerating myofibers can be visualized by their

135 characteristic feature of centrally located nuclei (46). Injured myofibers in this method were visually

136 selected by the presence of the centrally located myonuclei by Hoechst staining and the selected myofiber

137 was used for downstream processing with smfATAC-Seq (Figure 2A, 2B). The selection of a specific

138 myofiber that our smfATAC-Seq allows for, as well as the application of trypsin to remove any

139 confounding resident cell types present, results in the sequencing of DNA fragments corresponding purely

140 to the myonuclei within a specific myofiber.

141 smfATAC-Seq can identify the accessible chromatin regions of a single myofiber

142 To validate the quality of the ATAC-Seq data generated from a single EDL myofiber, we first

143 investigated the profiles of the ATAC-seq samples from both injured and uninjured myofibers as well as

144 freshly sorted MuSCs. We investigated the similarity between biological replicates for each condition

145 (i.e., uninjured and injured myofibers and MuSCs) by visualization of ATAC-Seq peaks for the muscle-

146 specific gene muscle creatine kinase $(\mathrm{Ckm})(47)$, the housekeeping gene Gapdh and the MuSC specific

147 gene myogenic factor 5 (Myf 5) (48). This analysis not only indicates that smfATAC-Seq can reliably

148 detect chromatin accessibility in a single myofiber but also the presence of comparable peaks in each

149 biological replicate within the specific condition shows the similarity between the samples (Supplemental

150 Figure 3A-C). In addition, Pearson correlation analysis between the biological replicates showed high

151 correlations of ATAC-seq reads between the replicates, indicating consistency within the samples

152 (Supplemental Figure 3D-J). Furthermore, we mapped ATAC-Seq peaks with DNase-Seq from skeletal

153 muscle (Sequence Read Archive, accession \# SRX191047) and the similarity between our ATAC-Seq and 
Sahinyan et al. 2021 manuscript

154 previous DNase-Seq was confirmed through the similar peaks present for representative genes, as

155 visualized on the IGV (Supplemental Figure 3A-C). These analyses suggest that smfATAC-Seq can

156 robustly measure chromatin accessibility and identify active cis-regulatory elements in a single EDL

157 myofiber.

158 Following the initial quality control and the correlation analysis, the biological replicates from the same 159 condition were pooled for further analysis. Peak annotation analysis for MuSCs revealed that more than

160 half of the peaks were in the intron/distal intergenic regions (i.e. enhancer regions) and about $25 \%$ of the

161 peaks were in the promoter region (Figure 2C, Supplemental Table 2). Peak annotations for the uninjured

162 and injured single myofibers also showed a great proportion of peaks in the enhancer and promoter

163 regions (Figure 2D,2E, Supplemental Table 2). In addition, an enrichment of ATAC-seq reads around

164 Transcription Start Sites (TSS) from all datasets was observed, which is a typical result expected from

165 ATAC-Seq (30) (Figure 2F-H).

166 To further assess the quality of the ATAC-Seq data, we analyzed select genes that are expressed by either

167 MuSCs or myofibers. For instance, in the myofiber samples, we confirmed the presence of ATAC-seq

168 peaks in the promoter regions of $\mathrm{Ckm}$, Actin alpha 1 (Actal), Myogenic factor 6 (Myf6) and Myosin

169 heavy chain 4 (Myh4), all of which are expressed by myofibers but not MuSCs $(14,47,49,50)$ (Figure 2I-

170 L). On the other hand, in MuSCs we observed peaks in the promoter regions of Paired box 7 ( $\operatorname{Pax} 7)$, and

$171 M y f 5$, genes that are known to be expressed in MuSCs $(48,51)$ (Figure 2M-N). Gapdh was used a

172 housekeeping gene for all samples (Figure 2O) and Pou5fl was used as a negative control (Figure 2P).

173 These observed peaks for known expressed genes demonstrate that our method, smf-ATAC-Seq can

174 reliably analyse chromatin accessibility in a single myofiber.

\section{Uninjured and injured myofibers and MuSCs display distinct chromatin states}

176 To show the global differences in chromatin accessibility between MuSCs, uninjured and injured

177 myofibers, we first performed heatmap clustering of Pearson correlation coefficients on all the

178 replicates/samples, which shows that the biological replicates within conditions are more similar to one

179 another than when compared to those from the other conditions (Figure 3A). This can also be observed

180 through Principal Component Analysis (PCA) where each condition clusters separately, with the injured

181 and uninjured myofibers being more similar to one another than to MuSCs (Figure 3B).

182 To ensure that the differences seen between myofibers were not due to differences in fiber types, we 183 investigated the chromatin accessibility of known marker genes for slow and fast fiber types. Troponin I2 
Sahinyan et al. 2021 manuscript

184 (Tnni2) and Troponin T3 (Tnnt3), markers of fast fiber types, (52,53) had a high level of chromatin

185 accessibility while Troponin T1 (Tnnt1) and Myosin heavy chain 7 (Myh7), expressed in slow fiber types

186 showed no chromatin accessibility $(53,54)$ (Supplemental Figure 4). This data indicates that only fast

187 fiber types were analyzed in this study and that the differences in the chromatin state between the injured

188 and uninjured myofibers were not due to the differences in the fiber types.

189 For further comparison of accessible regions between the conditions, we performed differential analysis

190 of ATAC-Seq peaks based on the regions defined by the consensus peak sets derived from the uninjured

191 and injured myofibers and MuSCs conditions. The clustering analysis based on the consensus peak set

192 shows the overall chromatin state differences between the MuSCs and injured and unjured myofibers

193 where the replicates of a condition are more similar to one another than to other conditions (Figure 3C).

194 The unique chromatin state in each condition can be can be observed through the pile up analysis that we

195 have performed for the more accessible peaks (LFC> 2) and less accessible peaks $(\mathrm{LFC}<0.5)$ between

196 uninjured myofibers and MuSCs as well as between uninjured and injured myofibers (Figure 3D). In

197 addition, the proportion of the differential peaks corresponding to various genomic regions such as

198 promoters and enhancers, differs depending on whether we compare MuSCs to myofibers or compare the

199 myofibers during regeneration and homeostasis. For instance, the differential peaks between uninjured

200 myofibers and MuSCs were mostly found close to the promoter region $(\leq 1 \mathrm{~kb})$ whereas in the uninjured

201 and injured myofibers comparison, a greater proportion of differential peaks were found in the

202 intron/distal intergenic regions (i.e. enhancer regions) (Figure 3E). This implies that MuSCs and

203 myofibers mostly differ in their promoter accessibility, whereas myofibers during homeostasis and

204 regeneration differ mostly at the level of distal regulatory elements.

205 Furthermore, we performed occupancy analysis (using DiffBind) in order to determine the unique and

206 common peaks for each comparison between the conditions. Since the occupancy analysis relies on the

207 peak score, the distribution pattern of the peak scores for all the conditions was assessed and found

208 similar despite the differences in the total number of peaks between the conditions (Supplemental Figure

209 5A). Occupancy analysis between uninjured myofibers and MuSCs revealed that MuSCs have 45533

210 unique peaks while myofibers contain only 535 unique peaks which are not present in MuSCs (Figure

211 3F). There are also many common accessible regions as seen from 5930 peaks that are common to both

212 uninjured myofibers and MuSCs. This analysis suggests that myonuclei share large number of open

213 chromatin with their parental stem cells. On the other hand, the occupancy analysis between uninjured

214 and injured myofibers revealed that there are 6352 overlapping peaks between the regenerating and

215 resting myofibers. However, this analysis also revealed that there are 15352 unique peaks in the injured 
Sahinyan et al. 2021 manuscript

216 myofibers and only 83 peaks that are unique to the resting myofiber (Figure 3F). Furthermore, when

217 comparing the read count between conditions around the center of unique peaks, it can be observed that

218 each condition displays a unique open chromatin signature (Supplemental Figure 5B, 5C).

\section{Comparative analysis of the chromatin state between MuSCs and myofibers}

220 To get a better understanding of the functional differences in chromatin accessibility between MuSCs and 221 myofibers, we first performed Gene Ontology (GO Biological Process) analysis on the genes associated 222 with the nearest peaks from the uninjured myofiber and on the genes associated with the nearest unique 223 peaks in the myofiber compared to MuSCs. As expected, this revealed myofiber specific biological 224 processes such as myofiber structure and organization (Figure 4A and Supplemental Figure 6A). On the 225 other hand, GO term analysis on the genes associated with the nearest peaks from MuSCs as well as the 226 genes associated with the nearest unique peaks to MuSCs revealed biological processes such as adherens 227 junction organization, membrane permeability and regulation of notch signaling which play key roles in 228 MuSCs quiescence and function (55,56) (Figure 4B and Supplemental Figure 6B). The analysis above 229 also revealed that genomic regions that remain in an open chromatin state when muscle stem cells fully 230 differentiate into myofibers correspond to genes that are involved in processes such as mitochondrial 231 transport, regulation of transcription and regulation of metabolites and energy (Supplemental Figure 6C).

232 The changes in the chromatin state between MuSCs and myofibers can also be observed from the volcano 233 plots showing differential peaks between conditions labeled by their nearest gene (Figure 4D). For 234 instance, genomic regions associated with genes such as muscle-specific titin-capping protein (Tcap), a 235 component of the skeletal muscle z-disc, as well as genes that are involved in regulatory and structural 236 functions in skeletal muscle such as titin gene (Ttn) are associated with more accessible chromatin regions 237 in the myofiber compared to MuSCs $(57,58)$ (Figure 4D).

238 Additionally, we performed GO term analysis between uninjured and injured myofibers, which revealed 239 that the accessible regions in the resting and regenerating myofibers corresponded to genes involved in 240 similar processes. GO term analysis on the genes associated with the nearest peaks from uninjured 241 myofibers and from the injured myofibers as well as the genes associated with the nearest common peaks 242 between uninjured and injured myofibers revealed biological processes involved in striated muscle cell 243 development, actomyosin structure and sarcomere organization, which are important for myofiber 244 structural formation and for the proper function of myofibers (Figure 4A, 4C, Supplemental Figure 6D). 245 On the other hand, genes associated with the nearest unique peaks from the injured myofibers mostly 246 belong to processes involved in structural components of the myofiber while the genes associated with the 
Sahinyan et al. 2021 manuscript

247 nearest unique peaks from uninjured myofibers correspond to genes involved in ion transport and

248 metabolism (Supplemental Figure 6E-F).

249 Identification of cell type specific pathways by global analysis of chromatin accessibility

250 To further understand the functional differences in chromatin accessibility between different cell types,

251 we investigated the cell-type-specific pathways. To accomplish this, Gene Set Enrichment Analysis

252 (GSEA) was performed on genes associated with differentially accessible peaks between the conditions.

253 Importantly, the GSEA between uninjured and injured myofibers revealed that inflammatory response

254 and Il2-Stat5 signaling and injury related pathways are still operational even after seven days of CTX-

255 mediated injury to muscle (59) (Figure 5A).

256 The GSEA between uninjured myofibers and MuSCs revealed that one of the top significantly enriched

257 pathways is myogenesis where we can observe that genes associated with differentiation and myofiber

258 function such as Myf6, Ckm and Tropomyosin 2 (Tpm2) $(14,47,60,61)$ have higher accessibility in the

259 myofiber compared to MuSCs (Figure 5B- 5D). On the other hand, genes associated with quiescence and

260 MuSCs such as alpha 7 integrin (Itga7) and Gpx3 are more accessible in MuSCs compared to the

261 myofiber, as expected (62,63) (Figure 5C, 5E).

263 Moreover, differential chromatin accessibility between the MuSCs and their myofiber derivatives show

264 differences in pathways that are known to be important for muscle, such as Notch and TGF $\beta$ signalling

$265(55,56,64,65)$ (Supplemental Figure 7). For example, increased accessibility of Notch1 is seen in MuSCs

266 while increased accessibility of Jagged-2 (Jag2) is observed in the myofibers regardless of whether they

267 are regenerating or homeostatic as seen by the height of the peaks at their promoters (Supplemental

268 Figure 7A-C). On the other hand, for TGF $\beta$ signalling, Noggin (Nog) shows more accessibility in the

269 myofibers while bone morphogenetic protein-4 (Bmp4) has increased accessibility in MuSCs

270 (Supplemental Figure 7D-F). Taken together, this data shows that smfATAC-Seq is an effective method

271 to analyze chromatin accessibility and to identify active cis-regulatory elements in a single muscle fiber

272 as well as to compare muscle fibers under different physiological conditions.

\section{DISCUSSION}

274 Analysis of the myofiber-specific chromatin state and gene expression profile is very limited due to the

275 heterogenous nature of muscle with the presence of numerous non-myogenic cells in the tissue. Whole

276 muscle or muscle biopsies represents a pooled result of numerous cell types which are present in the 
Sahinyan et al. 2021 manuscript

muscle tissue. To overcome this limitation, single nucleus RNA-Seq (snRNA-Seq) and ATAC-Seq

(snATAC-Seq) have been developed and performed on myonuclei to allow for the computational removal of other cell types (16-18). However, these methods still sequence all myonuclei present in the muscle and cannot distinguish between different myofibers within a muscle. Although snATAC-Seq provides high dimensionality, it is limited in sequencing depth due to the generation of sparse reads. Although computational pseudo bulking of snATAC-Seq can increase read numbers for comparative analysis between samples and conditions, the pooled reads represent average of all myonuclei within the sample.

In this study we have introduced a highly effective protocol based on adaption of OMNI ATAC-Seq (27) to quantify chromatin accessibility of a single EDL myofiber with high resolution and sequencing depth. This method allows comparative analysis of chromatin accessibility within and between muscle types with a potential wide-spread use in future studies to investigate fiber-specific epigenetic alterations in skeletal muscle.

The smfATAC-Seq protocol that we introduce in this study investigates the open chromatin state of myofibers at a single myofiber resolution, and with a high sequencing depth that allows for peak calling and differential peak analysis. Using this method, we have demonstrated that accessible chromatin regions of myonuclei contained within a single EDL myofiber can be tagmented and that high-quality sequencing ready libraries can be generated from these fragments. Sequencing of these libraries allow for sufficient depth and peak calling that can be used for genome-wide analysis of chromatin accessibility between myofibers. In this study, we have also demonstrated that smfATAC-Seq strictly investigates a single myofiber without the confounding presence of muscle resident, non-myogenic cell types. Additionally, application of trypsin to isolated myofibers effectively removes MuSCs that are associated with myofibers (66) and was confirmed by the absence of peaks at the promoters of known genes associated with muscle stem and niche cells. Although all of the smfATAC-Seq samples sequenced in this study were fast type myofibers, this protocol can be used to distinguish between different fiber types which could be applied to study myofiber heterogeneity under various physiological conditions. smfATAC-Seq peaks associated with genes involved in muscle structure and function such as Actal, Ckm and the myosin heavy chain cluster, indicates that our smfATAC-Seq is a robust technique to investigate genome-wide chromatin accessibility of a single myofiber.

308 A key implication of this technique is its applicability to the study of changes in chromatin accessibility 309 between myofibers in different contexts. As a demonstration of this, we have performed smfATAC-Seq 310 on uninjured myofibers as well as injured myofibers isolated seven days post-injury to investigate the 
Sahinyan et al. 2021 manuscript

311 changes in chromatin accessibility that occurs during regeneration. Through Pearson correlations and

312 PCA analysis, we showed that each cell type including resting and injured myofibers cluster separately,

313 indicating the power of smfATAC-Seq to determine chromatin signature from minute materials such as a

314 single myofiber. In addition, through occupancy analysis, we showed that there is a large difference in the

315 number of unique peaks present in the injured myofibers compared to uninjured myofibers. This indicates

316 that there are major modifications to chromatin accessibility in the context of regeneration. However, GO

317 term analysis of genes associated with the accessible chromatin regions in both injured and uninjured

318 fibers display significant similarities in the biological processes and pathways such as striated muscle cell

319 development, actomyosin structure and sarcomere organization, which are key factors for the proper

320 structure and function of muscle. Despite these similarities, there are certain trends in which uninjured

321 myofibers have increased accessibility in genes involved in energy metabolism, while injured myofibers

322 have greater accessibility in genes involved in myogenesis and inflammatory response which is what

323 would be expected in the case of an injury and regeneration (67). Despite the increase in chromatin

324 accessibility during injury, the accessible chromatin regions in both injured and uninjured fibers are

325 associated with genes involved in similar biological processes. This similarity at the gene network despite

326 differences in chromatin profile may suggest activation of multiple enhancers on core muscle structural

327 genes in the case of injury. Another possible reason could be the length of the recovery time where at

328 seven days post injury, a significant portion of early activated genes may have gone back to steady state.

329 Previously, a study investigating the changes in the transcriptional profile of MuSCs and various muscle

330 resident cells throughout different time points of muscle injury using single cell RNA-Seq, revealed that

331 after seven days of regeneration most cell types returned to a state that was similar to homeostasis (37).

332 Therefore, it is possible that harvesting the injured EDL myofibers seven days post injury allowed these

333 myofibers to return to a state reminiscent of homeostatic myofibers. smfATAC-Seq can be further

334 implemented to study the effects of regeneration on chromatin accessibility at different time points.

335 Further, our analyses of the myofibers in this study indicates that this technique can effectively compare

336 samples between conditions and could see future use in the study of chromatin accessibility of fibers

337 under different biologically relevant conditions.

Lastly, we have also used smfATAC-Seq to compare changes in chromatin accessibility between MuSCs

340 and myofibers. Our data shows that the regions of open chromatin in the myofibers correspond to genes

341 involved in structural components of the muscle, such as the z-disc, which are important for the proper

342 functioning of the muscle. On the other hand, open regions of chromtain in the MuSCs mostly correspond

343 to genes involved in membrane permeability, adherens junction organization and signalling pathways

344 implicated in the regulation of MuSC function (68). The analysis also revealed that chromatin regions that 
Sahinyan et al. 2021 manuscript

are accessible in both MuSCs and myofibers correspond to genes that are crucial for general function of cells such as those involved in mitochondrial transport, regulation of transcription and regulation of metabolites and energy.

Overall, smfATAC-Seq is a robust molecular tool that can be used to analyze genome-wide chromatin accessibility of a single myofiber. The sequencing depth from this approach, allows for in depth analysis, peak calling, quantitative analysis of chromatin accessibility and to identify active enhancers and

352 promoters in a single muscle fiber. smfATAC-Seq can be used to study epigenetic alterations that occur 353 in muscle fibers during development, and in response to exercise.

\section{MATERIALS AND METHODS}

\section{ATAC-Seq on a Single Myofiber}

\section{Isolation of Extensor Digitorum Longus (EDL) from cardiotoxin-induced injured muscle}

The Extensor Digitorum Longus (EDL) muscle was injured by intramuscular injection of $50 \mu \mathrm{L}$ of $5 \mu \mathrm{M}$ cardiotoxin (CTX) (Sigma, 11061-96-4). Mice were treated with carprofen 20 minutes prior to CTX injection and were injected with CTX under anesthesia by isoflurane. Mice were sacrificed seven days post injury and the EDL was collected from the hind limb of each mouse with the contra lateral EDL being used for the isolation of uninjured myofibers.

\section{Dissection of EDL muscle}

The EDL muscle was dissected as previously described (66). Briefly, the skin of the hindlimb was removed and the tibialis anterior (TA) muscle was excised with a pair of dissection scissors. The tendons of the EDL were exposed and cut from tendon to tendon with scissors.

\section{Isolation of a single EDL myofiber}

369 Individual myofibers were isolated from the EDL muscle as previously described (66). Briefly, the intact

370 EDL muscle was placed in a $1.5 \mathrm{~mL}$ eppendorf tube with $800 \mu \mathrm{L}$ of myofiber digestion buffer containing

$3711000 \mathrm{U} / \mathrm{mL}$ of collagenase from Clostridium histolyticum (Sigma, C0130) in un-supplemented DMEM

372 (Gibco, 11995-065) for 1 hour. Trypsin was added to the myofiber digestion buffer at a final

373 concentration of $0.25 \%$ to remove the myofiber associated muscle stem cells. The EDL myofibers were

374 then transferred into $2 \mathrm{~mL}$ of $1 \mathrm{X}$ PBS (Wisent, 311-425-CL) in a 6 well-plate that had previously been 
Sahinyan et al. 2021 manuscript

coated with DMEM supplemented with $10 \%$ horse serum (HS) (Wisent, 065250). The EDL was then gently pipetted up and down with a large-bore glass pipette to disassociate the myofibers.

\section{Selection of injured and uninjured myofibers}

Live myofibers in the 6-well plate were stained with $2 \mu \mathrm{L}$ of $5 \mathrm{mg} / \mathrm{mL}$ of Hoechst (Molecular Probes, $\mathrm{H} 1399)$ in $2 \mathrm{~mL}$ of $1 \mathrm{X}$ PBS for 5 minutes in a $37{ }^{\circ} \mathrm{C}$ with $5 \% \mathrm{CO}_{2}$ incubator. The myofibers were then visualized under a microscope and selected based on the myonuclei location, where myofibers with a pattern of centrally located nuclei were determined to be regenerating and picked for the injury condition. Individual myofibers were then transferred to $0.2 \mathrm{~mL}$ microtubes using a small-bore glass pipette coated with HS.

\section{Lysis and permeabilization of the myofiber}

Residual media was removed with pipette under a microscope. Individual myofibers in $0.2 \mathrm{~mL}$ microtubes were put in $10 \mu \mathrm{L}$ of $\mathrm{ddH}_{2} \mathrm{O}$ for 5 minutes on ice. The $\mathrm{ddH}_{2} \mathrm{O}$ was removed with a pipette under a microscope, ensuring that the myofiber remained in the tube. The myofiber was then permeabilized with $20 \mu \mathrm{L}$ of $0.5 \%$ Triton X-100 (Sigma, T9284) in PBS for 15 minutes at room temperature (RT). The permeabilization buffer was removed with a pipette under a microscope and the myofiber was washed twice with $200 \mu \mathrm{L}$ of $1 \mathrm{X}$ PBS.

\section{Tagmentation of the myofiber by $\operatorname{Tn} 5$ transposase}

395 Transposition and ATAC-seq library preparation for a single myofiber was adapted from previously described OMNI ATAC-Seq protocol (27). The permeabilized myonuclei were tagmented with tagmentation mixture optimized for use on a myofiber (20 $\mu \mathrm{L}$ Tagment DNA Buffer (TD Buffer) (Illumina, 20034197), 13.3 $\mu$ L PBS, 0.2\% Tween-20 (Sigma, P1379-1L), 0.02\% Digitonin (Promega, G9441), $1.39 \mu \mathrm{L}$ Tn5 (Illumina, 20034197) and $4.61 \mu \mathrm{L}$ water). Each single myofiber was incubated with $6 \mu \mathrm{L}$ of the tagmentation mixture at $37^{\circ} \mathrm{C}$ for 56 minutes with periodic shaking of the tubes every $5-7$ minutes. Following the transposition with Tn5, DNA was purified using a QIAquick PCR Purification Kit (Qiagen, 28104) according to the manufacturer's guidelines.

\section{Library preparation}

405 The purified DNA was PCR amplified for 15 cycles using Q5 High Fidelity DNA polymerase (New 406 England Biolabs, M0491S) with the incorporation of Illumina Nextera XT adaptors (Illumina, FC-131407 1001). The libraries were then size selected with AmpureXP Beads (Beckman, Cat\# A63880) at a 1: 0.85 408 ratio $(\mathrm{v} / \mathrm{v})$. The size selected libraries were verified for quality control by bioanalyzer as well as 
Sahinyan et al. 2021 manuscript

verification of the library size via visualization on an agarose gel stained with GelGreen dye (Biotium, 41005). Libraries were then sequenced on NovaSeq6000 Sprime Paired End (PE) 150 bp.

\section{ATAC-Seq on MuSCs}

\section{Isolation of MuSCs by fluorescence-activated cell sorting (FACS) for ATAC-Seq}

413 MuSCs were isolated by Fluorescence Activated Cell Sorting (FACS) as previously described (69).

414 Briefly, hindlimb muscles from Pax7/GFP ${ }^{+}$mice were dissected and chopped. The minced muscles were

415 then transferred into a $15 \mathrm{~mL}$ Falcon tube and digested in un-supplemented F10 media (Gibco, 11550043)

416 with $2.4 \mathrm{U} / \mathrm{mL}$ Collagenase D (Roche, 11088882001), 12 U/mL Dispase II (Roche, 39307800) and

$4170.5 \mathrm{mM} \mathrm{CaCl}_{2}$. Digestion was performed on a shaker in an incubator at $37{ }^{\circ} \mathrm{C}$ with $5 \% \mathrm{CO}_{2}$ for 30

418 minutes. Following the first digestion, digested muscles were centrifuged at $600 \mathrm{~g}$ for 20 seconds and the

419 supernatant was transferred to a $50 \mathrm{~mL}$ Falcon tube with 9 mL FBS (Wisent, 080450) and was kept on

420 ice. The remaining pellet was triturated and was digested for another 15 minutes with additional digestion

421 buffer added. After the final digestion, the digested muscle mixture was transferred to the $50 \mathrm{ml}$ Falcon

422 tube containing the previously digested mixture. The digested muscle mixture was then filtered through a

$42340 \mu \mathrm{m}$ cell strainer (Falcon, C352340) and was centrifuged at $600 \mathrm{~g}$ for 18 minutes at $4^{\circ} \mathrm{C}$. The pelleted

424 cells were then resuspended in $800 \mu \mathrm{L}$ FACS buffer that is composed of $2 \%$ FBS/ PBS (v:v), $0.5 \mathrm{mM}$

425 EDTA (Invitrogen, AM9261) and with $0.5 \mu \mathrm{L}$ DAPI (5mg/mL) (Invitrogen, D3671). Resuspended cells

426 were then filtered through $40 \mu \mathrm{m}$ cell strainer and were transferred into polypropylene round-bottom

427 FACS compatible tubes (Falcon, 352063). MuSCs were sorted with a FACSAria Fusion cytometer (BD

428 Biosciences) based on negative selection for DAPI and positive selection for GFP.

429 Lysis and transposition of MuSCs

430 ATAC-Seq on MuSCs was performed based on the previously established OMNI-ATAC-Seq protocol

431 (27). Briefly, five thousand MuSCs were sorted by FACS into $30 \mu \mathrm{L}$ of the ATAC lysis buffer containing $43210 \mathrm{mM}$ Tris-HCl (pH 7.5), 10 mM NaCl (Bioshop, 7647-14-5), 3 mM MgCl2 (Sigma, 7786-30-3), 0.1\%

433 Tween-20 (Sigma, P1379-1L), 0.1\% NP-40 (Sigma, 74385), and 0.01\% Digitonin (Promega, G9441) in a

$4340.2 \mathrm{~mL}$ microtube. Cells were incubated in the lysis buffer for 5 minutes on ice and then 3 minutes at 435 room temperature (RT). Cells were then washed with $100 \mu \mathrm{L}$ of wash buffer composed of $10 \mathrm{mM}$ Tris-

$436 \mathrm{HCl}(\mathrm{pH} 7.5), 10 \mathrm{Mm} \mathrm{NaCl}, 3 \mathrm{mM} \mathrm{MgCl} 2$ and $0.1 \%$ Tween-20, and were centrifuged at $800 \mathrm{~g}$ for 10

437 minutes. The pellet was resuspended in $10 \mu \mathrm{L}$ of transposition mixture $(5 \mu \mathrm{L}$ TD buffer, $3.2 \mu \mathrm{L}$ PBS, 0.89

$438 \mu \mathrm{L}$ Tn5 (Illumina, 20034197), 0.1\% Tween-20, 0.01\% Digitonin and $0.75 \mu \mathrm{L}$ nuclease free water).

439 Transposition was performed for 20 minutes at $37{ }^{\circ} \mathrm{C}$ while shaking the tubes every 5-7 minutes. The 
Sahinyan et al. 2021 manuscript

DNA was then purified using a QIAquick PCR Purification Kit according to the manufacturer's

441 guidelines.

\section{Library preparation for MuSCs ATAC-Seq}

444 The eluted tagmented DNA was PCR amplified for 12 cycles with the incorporation of Illumina Nextera 445 XT adapters using Q5 High Fidelity DNA polymerase. The libraries were then size selected with 446 AmpureXP Beads at a 1: 0.85 ratio (v/v). The libraries were then verified by bioanalyzer and agarose gel 447 visualization. Finally, the samples were sequenced on NovaSeq6000 Sprime Paired End (PE) 150 bp. ATAC-Seq data processing

The sequencing data was processed using the GenPipes pipeline v.3.1.5 (70). The raw reads were trimmed using Trimmomatic v.0.36 (71) and aligned to the mm10 genome assembly using the BurrowsWheeler Aligner v.0.7.12 (72). Reads were filtered to keep only high quality alignments (MAPQ score > 20) and duplicates were removed using SAMtools v.1.3.1 (73). Peak calling was performed with MACS2 v.2.1.1 (74) using piling up of paired-end fragment mode (--format BAMPE). The peak files (bed) were filtered by removing the ENCODE black listed regions

455 (https://www.encodeproject.org/files/ENCFF547MET) using BEDTools v2.29.1 (75). Mitochondrial 456 reads were also removed before the analysis.

\section{Correlation analysis between the biological replicates and clustering}

In order to perform a quantitative comparison of the read counts within accessible regions, the overlapping peaks of all replicates were merged using BEDTools v2.29.1 (75). This set of merged peaks and the BAM alignment files were used as input for the featureCounts function of Rsubread v.2.2.6 (76) to generate a raw-count matrix. The raw counts were normalized by rlog transformation using DESeq2 (77) with respect to library size. Pearson correlation coefficients were calculated based on the normalized counts for each pairwise comparison. Principal component analysis (PCA) and hierarchical clustering were also performed to evaluate the similarity between the replicates.

\section{Peak annotation analysis}

466 For each condition, the BAM alignment files of the replicates were merged and peak calling was

467 performed with MACS2 v.2.1.1 (74). Peak sets for each condition were annotated using the ChIPseaker

468 v.1.24.0 (78) annotatePeak function, and the UCSC Genome Browser knownGene (mm10) table. 
Sahinyan et al. 2021 manuscript

\section{Obtaining coverage tracks}

The BAM alignment files were converted to bigWig format and normalized by scaling factor (-scaleFactor) with the deepTools v.2.5.0.1 (79) bamCoverage function.

\section{Enrichment of genomic signal around TSS}

475 The bigWig files and the TSS coordinates obtained from the UCSC Genome Browser knownGene

476 (mm10) table were used as input for the computeMatrix function of deepTools v.2.5.0.1 (79). This matrix

477 was used for plotHeatmap function to generate the heatmap.

\section{Identification of overlapping/unique accessible regions}

480 For each comparison between the conditions, overlapping and unique accessible regions were identified with DiffBind v.2.16.2(80) based on the measure of confidence in the peak call by MACS2 v.2.1.1 (74).

\section{Analysis of differentially accessible regions}

The identification of differentially accessible regions (DARs) between the conditions was done using DiffBind v.2.16.2 (80) and edgeR v.3.30.1 (81). Log fold changes were calculated, and their associated pvalues were corrected for multiple hypothesis testing via the Benjamini-Hochberg procedure to obtain adjusted p-values. The DARs were annotated by their nearest gene using the annotatePeaks.pl function of Homer v.4.11(82).

\section{Gene set enrichment analysis}

Genes nearby the DARs were ranked based on the log-fold change calculated with edgeR v.3.30.1 (81).

494 This ranked list of genes was used as input to perform gene set enrichment analysis with the

495 fgseaMultilevel function of the R package fgsea v.1.14.0 (83). The FGSEA-multilevel method is based on 496 an adaptive multi-level split Monte Carlo scheme, which allows the estimation of very low p-values. The 497 Hallmark gene sets collection from the Molecular Signatures Database (MSigDB) (83) was used as a 498 reference to identify the biological processes that were significantly enriched.

\section{Animal care}

501 All procedures that were performed on animals were approved by the McGill University Animal Care 502 Committee (UACC). 
Sahinyan et al. 2021 manuscript

504 The data discussed in this study have been deposited in NCBI's Gene Expression Omnibus and are

505 accessible through GEO Series accession numbers GSE173676 and GSE171534.

\section{Author contributions}

Conceptualization: VDS; Methodology: KS, DMB, VDS; Investigation: VDS, MMS, KS, DMB, TK;

Visualization: KS, VDS, MMS, DMB; Funding acquisition: VDS; Project administration: VDS;

Supervision: VDS, GB; Software: MMS; Writing - original draft: KS, DMB; Writing - review \& editing: KS, DMB, VDS, MMS, FL.

\section{Acknowledgments}

515 We thank Christian Young at the Lady Davis Institute for Medical Research—Jewish General Hospital-

516 core facility for his help with fluorescence-activated cell sorting (FACS) of muscle stem cells. We thank

517 Dr. Michael Witcher at McGill University Department of Oncology for his critical comments and careful review of an early draft of this manuscript.

\section{Funding}

521 This work was supported and by discovery grant from Natural Sciences and Engineering Research

522 Council (NSERC) to VDS.

\section{Declaration of Interests}

525 The authors declare no competing interests.

\section{References}

1. Buckingham, M., Bajard, L., Chang, T., Daubas, P., Hadchouel, J., Meilhac, S., Montarras, D., Rocancourt, D., and Relaix, F. (2003) The formation of skeletal muscle: from somite to limb. J Anat 202, 59-68

2. Huxley, H., and Hanson, J. (1954) Changes in the cross-striations of muscle during contraction and stretch and their structural interpretation. Nature 173, 973-976

3. Zierath, J. R., and Hawley, J. A. (2004) Skeletal muscle fiber type: influence on contractile and metabolic properties. PLOS Biol 2, e348 stimulation of human muscle protein synthesis during continuous infusion of amino acids. J Physiol 532, 575-579 
Sahinyan et al. 2021 manuscript

5. Mittendorfer, B., Andersen, J. L., Plomgaard, P., Saltin, B., Babraj, J. A., Smith, K., and Rennie, M. J. (2005) Protein synthesis rates in human muscles: neither anatomical location nor fibre-type composition are major determinants. J Physiol 563, 203-211

6. Quiat, D., Voelker, K. A., Pei, J., Grishin, N. V., Grange, R. W., Bassel-Duby, R., and Olson, E. N. (2011) Concerted regulation of myofiber-specific gene expression and muscle performance by the transcriptional repressor Sox6. Proc Natl Acad Sci U S A 108, 1019610201

7. Weskamp, K., Olwin, B. B., and Parker, R. (2021) Post-Transcriptional Regulation in Skeletal Muscle Development, Repair, and Disease. Trends Mol Med 27, 469-481

8. Wilson, J. M., Loenneke, J. P., Jo, E., Wilson, G. J., Zourdos, M. C., and Kim, J. S. (2012) The effects of endurance, strength, and power training on muscle fiber type shifting. $J$ Strength Cond Res 26, 1724-1729

9. Dons, B., Bollerup, K., Bonde-Petersen, F., and Hancke, S. (1979) The effect of weightlifting exercise related to muscle fiber composition and muscle cross-sectional area in humans. Eur J Appl Physiol Occup Physiol 40, 95-106

10. Deschenes, M. R. (2004) Effects of aging on muscle fibre type and size. Sports Med 34, 809-824

11. Thompson, L. V. (2002) Skeletal muscle adaptations with age, inactivity, and therapeutic exercise. J Orthop Sports Phys Ther 32, 44-57

12. Nilwik, R., Snijders, T., Leenders, M., Groen, B. B., van Kranenburg, J., Verdijk, L. B., and van Loon, L. J. (2013) The decline in skeletal muscle mass with aging is mainly attributed to a reduction in type II muscle fiber size. Exp Gerontol 48, 492-498

13. Roberts, B. M., Frye, G. S., Ahn, B., Ferreira, L. F., and Judge, A. R. (2013) Cancer cachexia decreases specific force and accelerates fatigue in limb muscle. Biochem Biophys Res Commun 435, 488-492

14. Lazure, F., Blackburn, D. M., Corchado, A. H., Sahinyan, K., Karam, N., Sharanek, A., Nguyen, D., Lepper, C., Najafabadi, H. S., Perkins, T. J., Jahani-Asl, A., and Soleimani, V. D. (2020) Myf6/MRF4 is a myogenic niche regulator required for the maintenance of the muscle stem cell pool. EMBO Rep 21, e49499

15. Giordani, L., He, G. J., Negroni, E., Sakai, H., Law, J. Y. C., Siu, M. M., Wan, R., Corneau, A., Tajbakhsh, S., Cheung, T. H., and Le Grand, F. (2019) High-Dimensional Single-Cell Cartography Reveals Novel Skeletal Muscle-Resident Cell Populations. Mol Cell 74, 609621 e606

16. Petrany, M. J., Swoboda, C. O., Sun, C., Chetal, K., Chen, X., Weirauch, M. T., Salomonis, N., and Millay, D. P. (2020) Single-nucleus RNA-seq identifies transcriptional heterogeneity in multinucleated skeletal myofibers. Nat Commun 11, 6374

17. Dos Santos, M., Backer, S., Saintpierre, B., Izac, B., Andrieu, M., Letourneur, F., Relaix, F., Sotiropoulos, A., and Maire, P. (2020) Single-nucleus RNA-seq and FISH identify coordinated transcriptional activity in mammalian myofibers. Nat Commun 11, 5102

18. Kim, M., Franke, V., Brandt, B., Lowenstein, E. D., Schowel, V., Spuler, S., Akalin, A., and Birchmeier, C. (2020) Single-nucleus transcriptomics reveals functional compartmentalization in syncytial skeletal muscle cells. Nat Commun 11, 6375 
Sahinyan et al. 2021 manuscript

19. Zhu, F., Farnung, L., Kaasinen, E., Sahu, B., Yin, Y., Wei, B., Dodonova, S. O., Nitta, K. R., Morgunova, E., Taipale, M., Cramer, P., and Taipale, J. (2018) The interaction landscape between transcription factors and the nucleosome. Nature 562, 76-81

20. Thurman, R. E., Rynes, E., Humbert, R., Vierstra, J., Maurano, M. T., Haugen, E., Sheffield, N. C., Stergachis, A. B., Wang, H., Vernot, B., Garg, K., John, S., Sandstrom, R., Bates, D., Boatman, L., Canfield, T. K., Diegel, M., Dunn, D., Ebersol, A. K., Frum, T., Giste, E., Johnson, A. K., Johnson, E. M., Kutyavin, T., Lajoie, B., Lee, B. K., Lee, K., London, D., Lotakis, D., Neph, S., Neri, F., Nguyen, E. D., Qu, H., Reynolds, A. P., Roach, V., Safi, A., Sanchez, M. E., Sanyal, A., Shafer, A., Simon, J. M., Song, L., Vong, S., Weaver, M., Yan, Y., Zhang, Z., Zhang, Z., Lenhard, B., Tewari, M., Dorschner, M. O., Hansen, R. S., Navas, P. A., Stamatoyannopoulos, G., Iyer, V. R., Lieb, J. D., Sunyaev, S. R., Akey, J. M., Sabo, P. J., Kaul, R., Furey, T. S., Dekker, J., Crawford, G. E., and Stamatoyannopoulos, J. A. (2012) The accessible chromatin landscape of the human genome. Nature 489, 75-82

21. Liu, L., Leng, L., Liu, C., Lu, C., Yuan, Y., Wu, L., Gong, F., Zhang, S., Wei, X., Wang, M., Zhao, L., Hu, L., Wang, J., Yang, H., Zhu, S., Chen, F., Lu, G., Shang, Z., and Lin, G. (2019) An integrated chromatin accessibility and transcriptome landscape of human preimplantation embryos. Nat Commun 10, 364

22. Trevino, A. E., Sinnott-Armstrong, N., Andersen, J., Yoon, S. J., Huber, N., Pritchard, J. K., Chang, H. Y., Greenleaf, W. J., and Pasca, S. P. (2020) Chromatin accessibility dynamics in a model of human forebrain development. Science 367

23. Corces, M. R., Granja, J. M., Shams, S., Louie, B. H., Seoane, J. A., Zhou, W., Silva, T. C., Groeneveld, C., Wong, C. K., Cho, S. W., Satpathy, A. T., Mumbach, M. R., Hoadley, K. A., Robertson, A. G., Sheffield, N. C., Felau, I., Castro, M. A. A., Berman, B. P., Staudt, L. M., Zenklusen, J. C., Laird, P. W., Curtis, C., Cancer Genome Atlas Analysis, N., Greenleaf, W. J., and Chang, H. Y. (2018) The chromatin accessibility landscape of primary human cancers. Science $\mathbf{3 6 2}$

24. Rendeiro, A. F., Schmidl, C., Strefford, J. C., Walewska, R., Davis, Z., Farlik, M., Oscier, D., and Bock, C. (2016) Chromatin accessibility maps of chronic lymphocytic leukaemia identify subtype-specific epigenome signatures and transcription regulatory networks. Nat Commun 7, 11938

25. Wang, Y., Zhang, X., Song, Q., Hou, Y., Liu, J., Sun, Y., and Wang, P. (2020) Characterization of the chromatin accessibility in an Alzheimer's disease (AD) mouse model. Alzheimers Res Ther 12, 29

26. Bastle, R. M., and Maze, I. (2019) Chromatin Regulation in Complex Brain Disorders. Curr Opin Behav Sci 25, 57-65

27. Corces, M. R., Trevino, A. E., Hamilton, E. G., Greenside, P. G., Sinnott-Armstrong, N. A., Vesuna, S., Satpathy, A. T., Rubin, A. J., Montine, K. S., Wu, B., Kathiria, A., Cho, S. W., Mumbach, M. R., Carter, A. C., Kasowski, M., Orloff, L. A., Risca, V. I., Kundaje, A., Khavari, P. A., Montine, T. J., Greenleaf, W. J., and Chang, H. Y. (2017) An improved ATAC-seq protocol reduces background and enables interrogation of frozen tissues. Nat Methods 14, 959-962

28. Buenrostro, J. D., Wu, B., Chang, H. Y., and Greenleaf, W. J. (2015) ATAC-seq: A Method for Assaying Chromatin Accessibility Genome-Wide. Curr Protoc Mol Biol 109, 2129 21212929 
Sahinyan et al. 2021 manuscript

29. Liu, C., Wang, M., Wei, X., Wu, L., Xu, J., Dai, X., Xia, J., Cheng, M., Yuan, Y., Zhang, P., Li, J., Feng, T., Chen, A., Zhang, W., Chen, F., Shang, Z., Zhang, X., Peters, B. A., and Liu, L. (2019) An ATAC-seq atlas of chromatin accessibility in mouse tissues. Sci Data 6, 65

30. Yan, F., Powell, D. R., Curtis, D. J., and Wong, N. C. (2020) From reads to insight: a hitchhiker's guide to ATAC-seq data analysis. Genome Biol 21, 22

31. Rocks, D., Jaric, I., Tesfa, L., Greally, J. M., Suzuki, M., and Kundakovic, M. (2021) Cell type-specific chromatin accessibility analysis in the mouse and human brain. Epigenetics, 1-18

32. Janssen, I., Heymsfield, S. B., Wang, Z. M., and Ross, R. (2000) Skeletal muscle mass and distribution in 468 men and women aged 18-88 yr. J Appl Physiol (1985) 89, 81-88

33. Ortenblad, N., Nielsen, J., Boushel, R., Soderlund, K., Saltin, B., and Holmberg, H. C. (2018) The Muscle Fiber Profiles, Mitochondrial Content, and Enzyme Activities of the Exceptionally Well-Trained Arm and Leg Muscles of Elite Cross-Country Skiers. Front Physiol 9, 1031

34. Mishra, P., Varuzhanyan, G., Pham, A. H., and Chan, D. C. (2015) Mitochondrial Dynamics is a Distinguishing Feature of Skeletal Muscle Fiber Types and Regulates Organellar Compartmentalization. Cell Metab 22, 1033-1044

35. Neal, A., Boldrin, L., and Morgan, J. E. (2012) The satellite cell in male and female, developing and adult mouse muscle: distinct stem cells for growth and regeneration. PLoS One 7, e37950

36. Cramer, A. A. W., Prasad, V., Eftestol, E., Song, T., Hansson, K. A., Dugdale, H. F., Sadayappan, S., Ochala, J., Gundersen, K., and Millay, D. P. (2020) Nuclear numbers in syncytial muscle fibers promote size but limit the development of larger myonuclear domains. Nat Commun 11, 6287

37. De Micheli, A. J., Laurilliard, E. J., Heinke, C. L., Ravichandran, H., Fraczek, P., SoueidBaumgarten, S., De Vlaminck, I., Elemento, O., and Cosgrove, B. D. (2020) Single-Cell Analysis of the Muscle Stem Cell Hierarchy Identifies Heterotypic Communication Signals Involved in Skeletal Muscle Regeneration. Cell Rep 30, 3583-3595 e3585

38. De Micheli, A. J., Spector, J. A., Elemento, O., and Cosgrove, B. D. (2020) A reference single-cell transcriptomic atlas of human skeletal muscle tissue reveals bifurcated muscle stem cell populations. Skelet Muscle 10, 19

39. Khan, S. S., Solomon, M. A., and McCoy, J. P., Jr. (2005) Detection of circulating endothelial cells and endothelial progenitor cells by flow cytometry. Cytometry B Clin Cytom 64, 1-8

40. Steppan, C. M., Bailey, S. T., Bhat, S., Brown, E. J., Banerjee, R. R., Wright, C. M., Patel, H. R., Ahima, R. S., and Lazar, M. A. (2001) The hormone resistin links obesity to diabetes. Nature 409, 307-312

41. McKinney-Freeman, S. L., Naveiras, O., Yates, F., Loewer, S., Philitas, M., Curran, M., Park, P. J., and Daley, G. Q. (2009) Surface antigen phenotypes of hematopoietic stem cells from embryos and murine embryonic stem cells. Blood 114, 268-278

42. Agorku, D. J., Langhammer, A., Heider, U., Wild, S., Bosio, A., and Hardt, O. (2019) CD49b, CD87, and CD95 Are Markers for Activated Cancer-Associated Fibroblasts Whereas CD39 Marks Quiescent Normal Fibroblasts in Murine Tumor Models. Front Oncol 9, 716 
Sahinyan et al. 2021 manuscript

43. Waddell, L. A., Lefevre, L., Bush, S. J., Raper, A., Young, R., Lisowski, Z. M., McCulloch, M. E. B., Muriuki, C., Sauter, K. A., Clark, E. L., Irvine, K. M., Pridans, C., Hope, J. C., and Hume, D. A. (2018) ADGRE1 (EMR1, F4/80) Is a Rapidly-Evolving Gene Expressed in Mammalian Monocyte-Macrophages. Front Immunol 9, 2246

44. Joe, A. W., Yi, L., Natarajan, A., Le Grand, F., So, L., Wang, J., Rudnicki, M. A., and Rossi, F. M. (2010) Muscle injury activates resident fibro/adipogenic progenitors that facilitate myogenesis. Nat Cell Biol 12, 153-163

45. Folker, E. S., and Baylies, M. K. (2013) Nuclear positioning in muscle development and disease. Front Physiol 4, 363

46. Roman, W., and Gomes, E. R. (2018) Nuclear positioning in skeletal muscle. Semin Cell Dev Biol 82, 51-56

47. Tai, P. W., Fisher-Aylor, K. I., Himeda, C. L., Smith, C. L., Mackenzie, A. P., Helterline, D. L., Angello, J. C., Welikson, R. E., Wold, B. J., and Hauschka, S. D. (2011) Differentiation and fiber type-specific activity of a muscle creatine kinase intronic enhancer. Skelet Muscle 1, 25

48. Rudnicki, M. A., Schnegelsberg, P. N., Stead, R. H., Braun, T., Arnold, H. H., and Jaenisch, R. (1993) MyoD or Myf-5 is required for the formation of skeletal muscle. Cell 75, 13511359

49. Nowak, K. J., Wattanasirichaigoon, D., Goebel, H. H., Wilce, M., Pelin, K., Donner, K., Jacob, R. L., Hubner, C., Oexle, K., Anderson, J. R., Verity, C. M., North, K. N., lannaccone, S. T., Muller, C. R., Nurnberg, P., Muntoni, F., Sewry, C., Hughes, I., Sutphen, R., Lacson, A. G., Swoboda, K. J., Vigneron, J., Wallgren-Pettersson, C., Beggs, A. H., and Laing, N. G. (1999) Mutations in the skeletal muscle alpha-actin gene in patients with actin myopathy and nemaline myopathy. Nat Genet 23, 208-212

50. Stuart, C. A., Stone, W. L., Howell, M. E., Brannon, M. F., Hall, H. K., Gibson, A. L., and Stone, M. H. (2016) Myosin content of individual human muscle fibers isolated by laser capture microdissection. Am J Physiol Cell Physiol 310, C381-389

51. Seale, P., Sabourin, L. A., Girgis-Gabardo, A., Mansouri, A., Gruss, P., and Rudnicki, M. A. (2000) Pax7 is required for the specification of myogenic satellite cells. Cell 102, 777-786

52. Mullen, A. J., and Barton, P. J. (2000) Structural characterization of the human fast skeletal muscle troponin I gene (TNNI2). Gene 242, 313-320

53. Wei, B., and Jin, J. P. (2016) TNNT1, TNNT2, and TNNT3: Isoform genes, regulation, and structure-function relationships. Gene 582, 1-13

54. Meredith, C., Herrmann, R., Parry, C., Liyanage, K., Dye, D. E., Durling, H. J., Duff, R. M., Beckman, K., de Visser, M., van der Graaff, M. M., Hedera, P., Fink, J. K., Petty, E. M., Lamont, P., Fabian, V., Bridges, L., Voit, T., Mastaglia, F. L., and Laing, N. G. (2004) Mutations in the slow skeletal muscle fiber myosin heavy chain gene (MYH7) cause laing early-onset distal myopathy (MPD1). Am J Hum Genet 75, 703-708

55. Bjornson, C. R., Cheung, T. H., Liu, L., Tripathi, P. V., Steeper, K. M., and Rando, T. A. (2012) Notch signaling is necessary to maintain quiescence in adult muscle stem cells. Stem Cells 30, 232-242

56. Mourikis, P., and Tajbakhsh, S. (2014) Distinct contextual roles for Notch signalling in skeletal muscle stem cells. BMC Dev Biol 14, 2 
Sahinyan et al. 2021 manuscript

57. Markert, C. D., Meaney, M. P., Voelker, K. A., Grange, R. W., Dalley, H. W., Cann, J. K., Ahmed, M., Bishwokarma, B., Walker, S. J., Yu, S. X., Brown, M., Lawlor, M. W., Beggs, A. H., and Childers, M. K. (2010) Functional muscle analysis of the Tcap knockout mouse. Hum Mol Genet 19, 2268-2283

58. Hackman, P., Vihola, A., Haravuori, H., Marchand, S., Sarparanta, J., De Seze, J., Labeit, S., Witt, C., Peltonen, L., Richard, I., and Udd, B. (2002) Tibial muscular dystrophy is a titinopathy caused by mutations in TTN, the gene encoding the giant skeletal-muscle protein titin. Am J Hum Genet 71, 492-500

59. Laurence, A., Tato, C. M., Davidson, T. S., Kanno, Y., Chen, Z., Yao, Z., Blank, R. B., Meylan, F., Siegel, R., Hennighausen, L., Shevach, E. M., and O'Shea J, J. (2007) Interleukin-2 signaling via STAT5 constrains T helper 17 cell generation. Immunity 26, 371-381

60. Buckingham, M. (1994) Muscle differentiation. Which myogenic factors make muscle? Curr Biol 4, 61-63

61. Jin, Y., Peng, Y., Lin, Z., Chen, Y. C., Wei, L., Hacker, T. A., Larsson, L., and Ge, Y. (2016) Comprehensive analysis of tropomyosin isoforms in skeletal muscles by top-down proteomics. J Muscle Res Cell Motil 37, 41-52

62. El Haddad, M., Jean, E., Turki, A., Hugon, G., Vernus, B., Bonnieu, A., Passerieux, E., Hamade, A., Mercier, J., Laoudj-Chenivesse, D., and Carnac, G. (2012) Glutathione peroxidase 3 , a new retinoid target gene, is crucial for human skeletal muscle precursor cell survival. J Cell Sci 125, 6147-6156

63. Pasut, A., Oleynik, P., and Rudnicki, M. A. (2012) Isolation of muscle stem cells by fluorescence activated cell sorting cytometry. Methods Mol Biol 798, 53-64

64. Carlson, M. E., Conboy, M. J., Hsu, M., Barchas, L., Jeong, J., Agrawal, A., Mikels, A. J., Agrawal, S., Schaffer, D. V., and Conboy, I. M. (2009) Relative roles of TGF-beta1 and Wnt in the systemic regulation and aging of satellite cell responses. Aging Cell 8, 676689

65. Girardi, F., Taleb, A., Ebrahimi, M., Datye, A., Gamage, D. G., Peccate, C., Giordani, L., Millay, D. P., Gilbert, P. M., Cadot, B., and Le Grand, F. (2021) TGFbeta signaling curbs cell fusion and muscle regeneration. Nat Commun 12, 750

66. Blackburn, D. M., Lazure, F., Corchado, A. H., Perkins, T. J., Najafabadi, H. S., and Soleimani, V. D. (2019) High-resolution genome-wide expression analysis of single myofibers using SMART-Seq. J Biol Chem 294, 20097-20108

67. Tidball, J. G. (2011) Mechanisms of muscle injury, repair, and regeneration. Compr Physiol 1, 2029-2062

68. Relaix, F., Bencze, M., Borok, M. J., Der Vartanian, A., Gattazzo, F., Mademtzoglou, D., Perez-Diaz, S., Prola, A., Reyes-Fernandez, P. C., Rotini, A., and Taglietti, t. (2021) Perspectives on skeletal muscle stem cells. Nat Commun 12, 692

69. Tichy, E. D., Sidibe, D. K., Greer, C. D., Oyster, N. M., Rompolas, P., Rosenthal, N. A., Blau, H. M., and Mourkioti, F. (2018) A robust Pax7EGFP mouse that enables the visualization of dynamic behaviors of muscle stem cells. Skelet Muscle 8, 27

70. Bourgey, M., Dali, R., Eveleigh, R., Chen, K. C., Letourneau, L., Fillon, J., Michaud, M., Caron, M., Sandoval, J., Lefebvre, F., Leveque, G., Mercier, E., Bujold, D., Marquis, P., Van, P. T., Anderson de Lima Morais, D., Tremblay, J., Shao, X., Henrion, E., Gonzalez, E., 
Sahinyan et al. 2021 manuscript

Quirion, P. O., Caron, B., and Bourque, G. (2019) GenPipes: an open-source framework for distributed and scalable genomic analyses. Gigascience 8

71. Bolger, A. M., Lohse, M., and Usadel, B. (2014) Trimmomatic: a flexible trimmer for Illumina sequence data. Bioinformatics 30, 2114-2120

72. Li, H., and Durbin, R. (2009) Fast and accurate short read alignment with BurrowsWheeler transform. Bioinformatics 25, 1754-1760

73. Li, H., Handsaker, B., Wysoker, A., Fennell, T., Ruan, J., Homer, N., Marth, G., Abecasis, G., Durbin, R., and Genome Project Data Processing, S. (2009) The Sequence Alignment/Map format and SAMtools. Bioinformatics 25, 2078-2079

74. Zhang, Y., Liu, T., Meyer, C. A., Eeckhoute, J., Johnson, D. S., Bernstein, B. E., Nusbaum, C., Myers, R. M., Brown, M., Li, W., and Liu, X. S. (2008) Model-based analysis of ChIPSeq (MACS). Genome Biol 9, R137

75. Quinlan, A. R. (2014) BEDTools: The Swiss-Army Tool for Genome Feature Analysis. Curr Protoc Bioinformatics 47, 1112 11-34

76. Liao, Y., Smyth, G. K., and Shi, W. (2014) featureCounts: an efficient general purpose program for assigning sequence reads to genomic features. Bioinformatics 30, 923-930

77. Love, M. I., Huber, W., and Anders, S. (2014) Moderated estimation of fold change and dispersion for RNA-seq data with DESeq2. Genome Biol 15, 550

78. Yu, G., Wang, L. G., and He, Q. Y. (2015) ChIPseeker: an R/Bioconductor package for ChIP peak annotation, comparison and visualization. Bioinformatics 31, 2382-2383

79. Ramirez, F., Dundar, F., Diehl, S., Gruning, B. A., and Manke, T. (2014) deepTools: a flexible platform for exploring deep-sequencing data. Nucleic Acids Res 42, W187-191

80. Stark R, B. G. (2011) DiffBind: differential binding analysis of ChIP-Seq peak data. in Bioconductor

81. Robinson, M. D., McCarthy, D. J., and Smyth, G. K. (2010) edgeR: a Bioconductor package for differential expression analysis of digital gene expression data.

Bioinformatics 26, 139-140

82. Heinz, S., Benner, C., Spann, N., Bertolino, E., Lin, Y. C., Laslo, P., Cheng, J. X., Murre, C., Singh, H., and Glass, C. K. (2010) Simple combinations of lineage-determining transcription factors prime cis-regulatory elements required for macrophage and $\mathrm{B}$ cell identities. Mol Cell 38, 576-589

83. Korotkevich, G., Sukhov, V., Budin, N., Shpak, B., Artyomov, M. N., and Sergushichev, A. (2021) Fast gene set enrichment analysis. bioRxiv, 060012

84. Mclean, C. Y., Bristor, D., Hiller, M., Clarke, S. L., Schaar, B. T., Lowe, C. B., Wenger, A. M., and Bejerano, G. (2010) GREAT improves functional interpretation of cis-regulatory regions. Nat Biotechnol 28, 495-501 


\section{Figure 1: Schematic of ATAC-seq performed on a single myofiber}

Schematic showing the steps and reactions involved in the preparation of sequencing ready libraries of single myofiber DNA for ATAC-Seq. Briefly, myofibers were isolated from the EDL muscle and an individual myofiber was transferred to a $0.2 \mathrm{~mL}$ microtube. The myofiber was then lysed with $\mathrm{ddH}_{2} \mathrm{O}$ and the myonuclei were permeabilized with $0.5 \%$ Triton X-100. Then, open chromatin regions were tagmented with hyperactive Tn5 transposase and the DNA fragments were purified through column purification. The tagmented DNA was then amplified by PCR and Nextera adapters were incorporated. Finally, size selection and purification was performed using 0.85X AMPure beads, resulting in sequencing ready libraries. 
Figure 1

1. Myofiber isolation

2. Individual myofiber transfered to tube, lysed with water and permeabilized with $0.5 \%$ Triton-X

3. DNA tagmentation and adaptor incorporation

4. Column purification

5. $\mathrm{PCR}$ amplification and library preperation

6. AMPure bead size selelction and purification

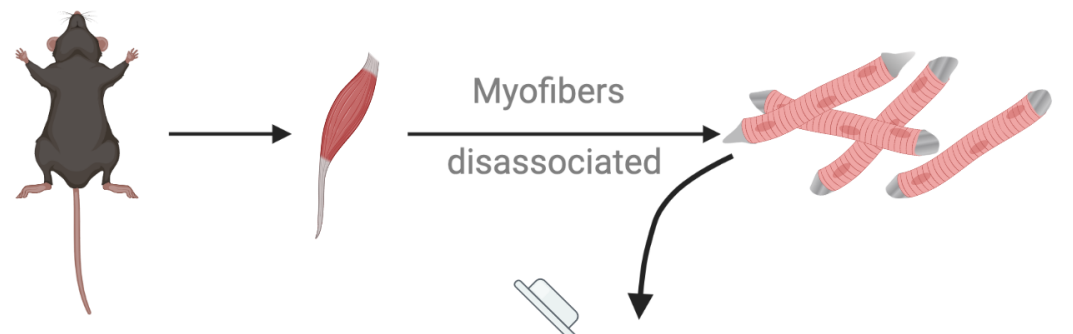

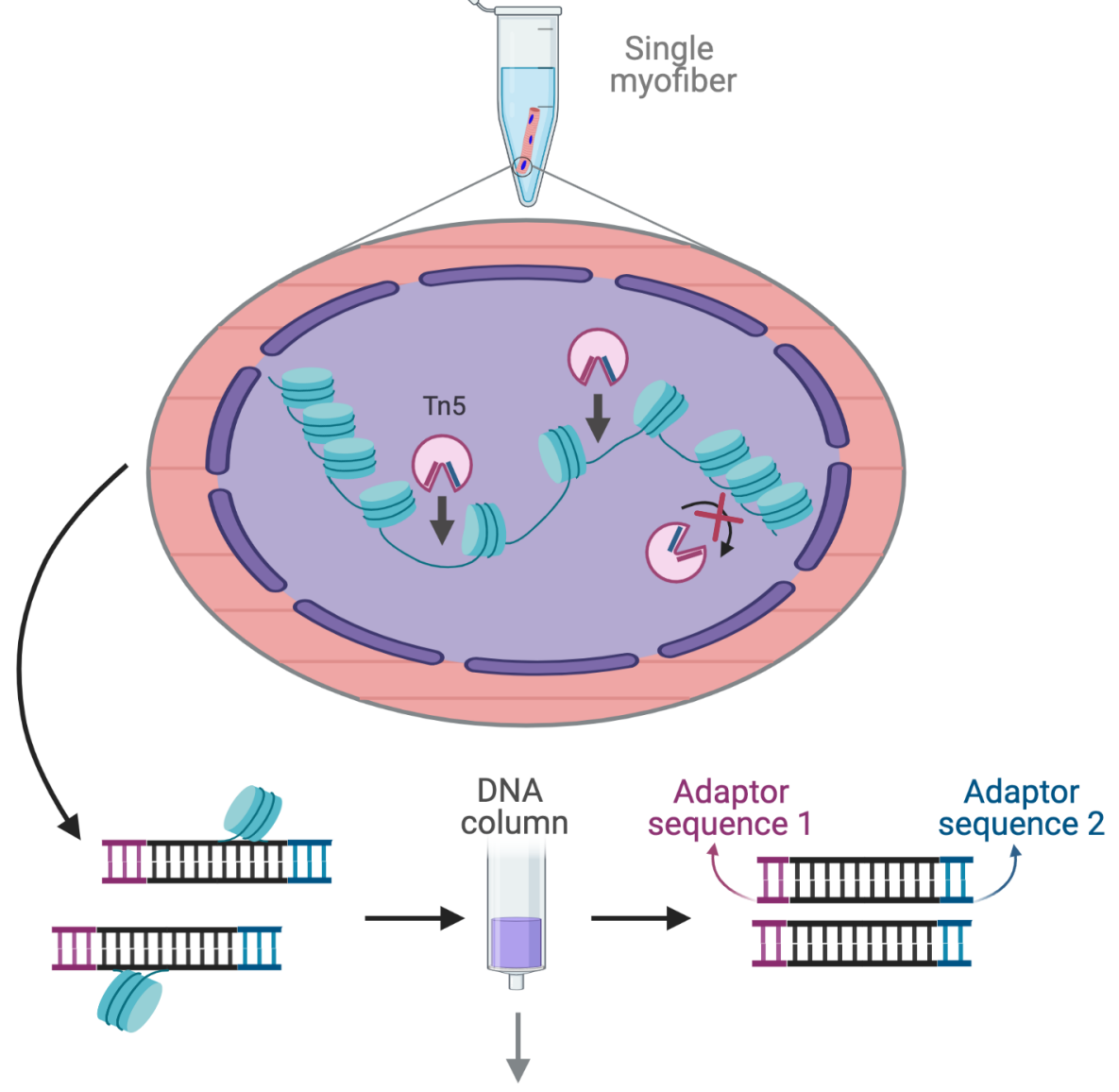

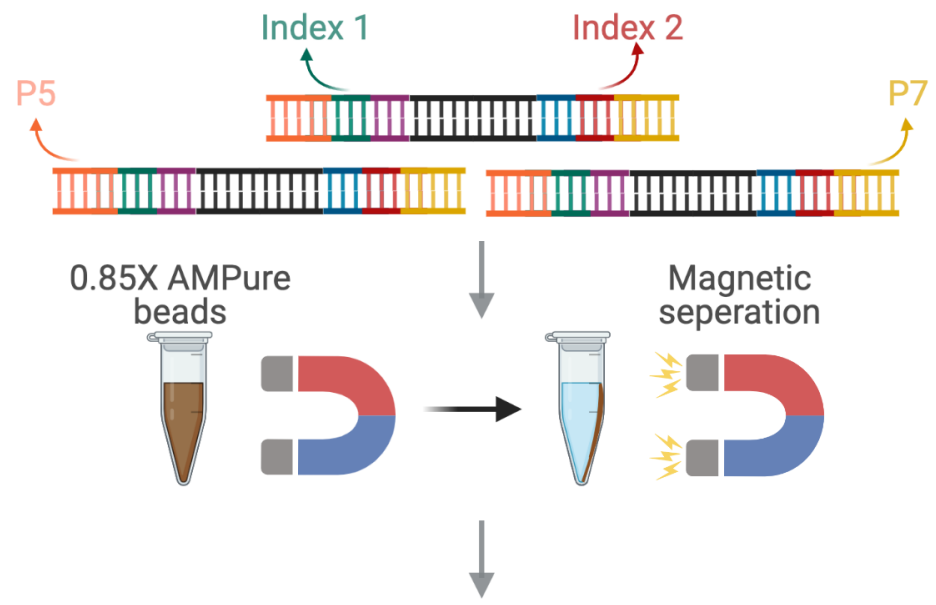

Sequence 
Figure 2: smfATAC-Seq can effectively identify the accessible regions on a single myofiber

(A) Representative picture of an isolated WT C57BL/6J uninjured myofiber stained for DAPI showing presence and location of myonuclei. Scale bar $=50 \mu \mathrm{m}$. (B) Representative picture of an isolated WT C57BL/6J injured myofiber (7 days after cardiotoxin induced injury) stained for DAPI showing centrally located myonuclei as a marker of a regenerating fiber. Scale bar $=50 \mu \mathrm{m}$. (C-E) Peak annotation pie charts for ATAC-Seq peaks of MuSCs, injured myofibers and uninjured myofibers, respectively. (F-H) Heatmaps showing enrichment at transcription start site (TSS) for the ATAC-Seq libraries of MuSCs, injured myofibers and uninjured myofibers, respectively. (I-P) IGV snapshots of known genes expressed in muscle fiber and/or MuSCs displaying accessibility on their respective TSS. (I) The muscle creatine kinase (Ckm). (J) Actin alpha 1 (Actal). (K) Part of the myosin heavy chain (Myh) gene cluster. (L) Myogenic factor 6 (Myf6). (M) Paired box 7 (Pax7). (N) Myogenic factor 5 (Myf5). (O) Housekeeping gene Gapdh. (P) POU Class 5 homeobox 1 (Pou5fl) as a negative control. 
Figure 2

bioRxiv preprint doi: https://doi.org/10.1101/2021.06.14.448380; this version posted June 15, 2021. The copyright holder for this preprint (which was not certified by peer review) is the author/funder.All rights reserved. No reuse allowed without permission.
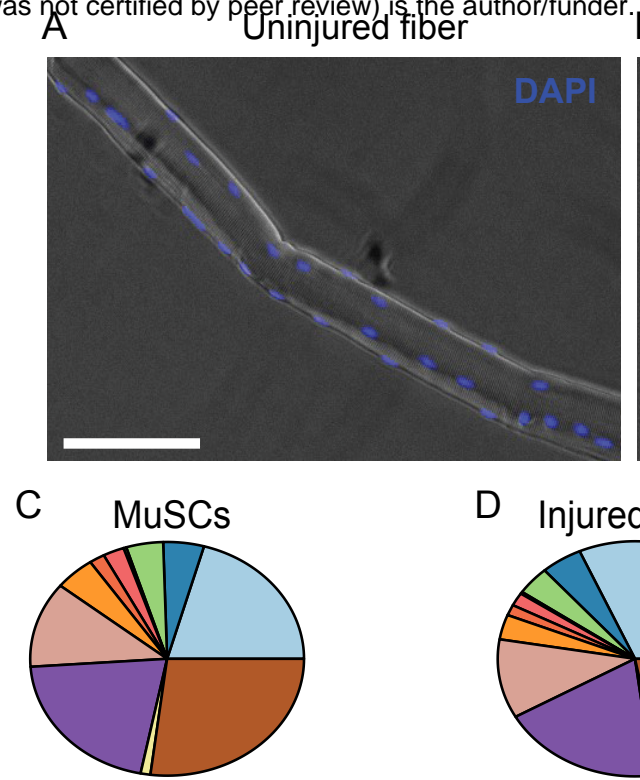

Promoter $(<=1 \mathrm{~kb})$ Promoter $(2-3 \mathrm{~kb})$

Promoter (1-2 kb) घ'UTR

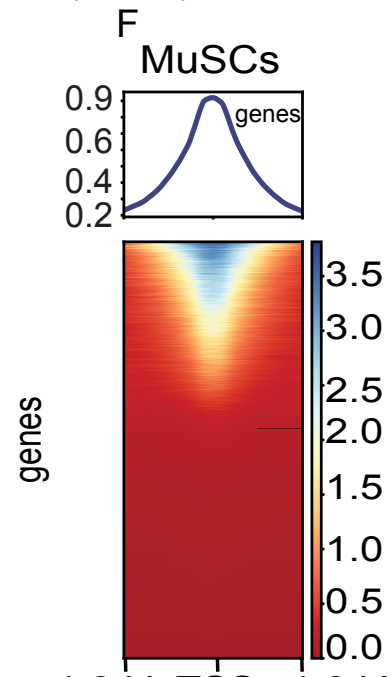

$-1.0 \mathrm{~kb}$ TSS $+1.0 \mathrm{~kb}$

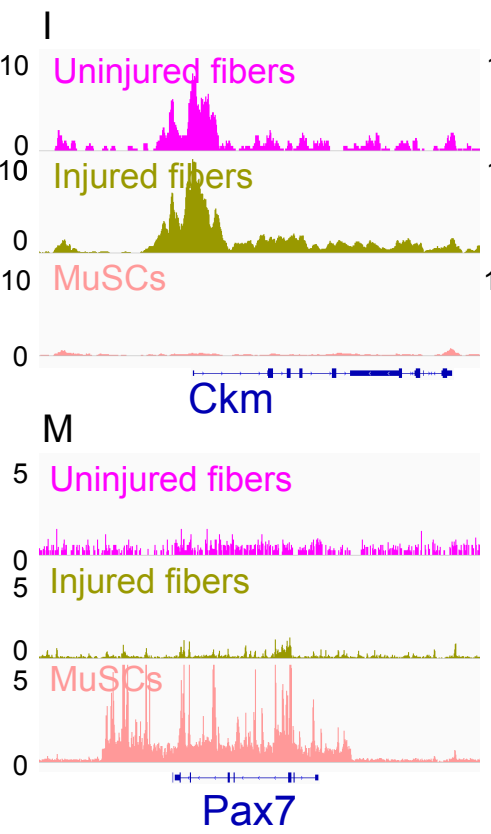
15 MuSCS $\mathrm{N}$ Acta1 5 Uninjured fibers
0

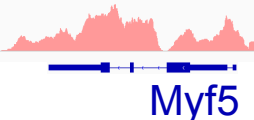

D Injured fibers

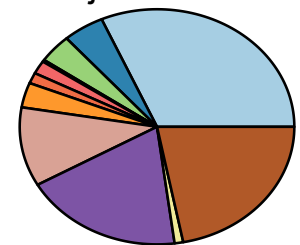

Other Exon 1st Exon 1st Intron G Injured fibers

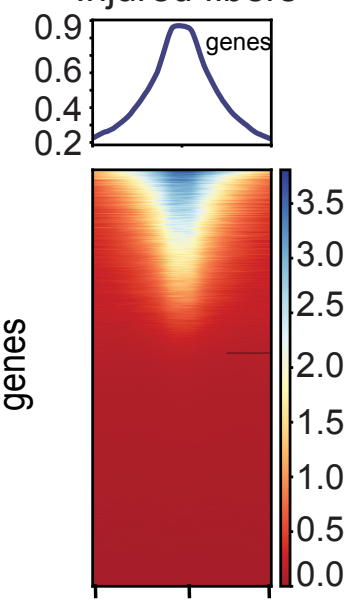

$-1.0 \mathrm{~kb}$ TSS +1.0 kb

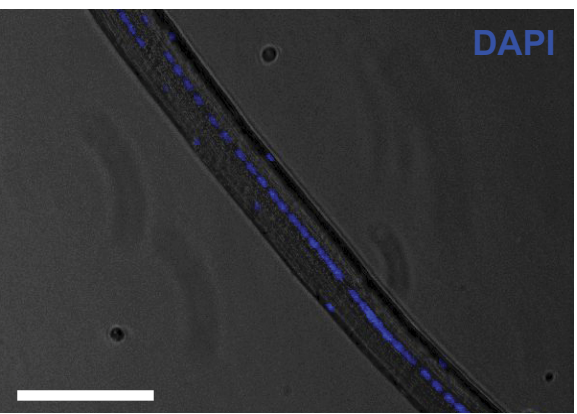

E Uninjured fibers

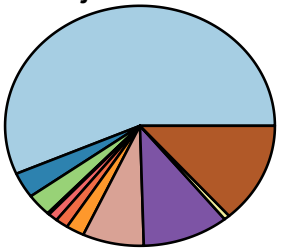

Other Intron Distal Intergenic

Downstream (<=300 kb)

$\mathrm{H}$

Uninjured fibers
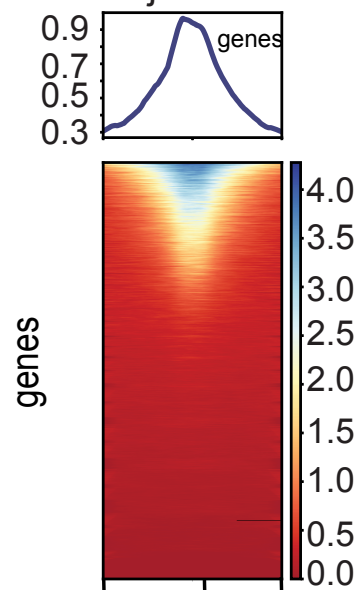

$-1.0 \mathrm{~kb}$ TSS +1.0 kb

L

5 Uninjured fipers

3 Uninjured fibers

0 3 Injured fibers 5 Injured fibers

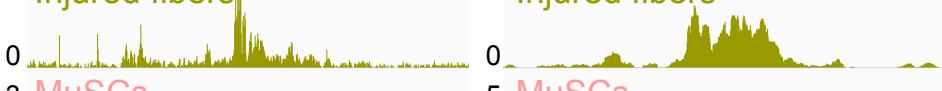
3 MuSCs 5 MuSCs

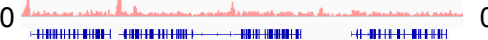
Myh2 Myh1 Myh4 Myh8 10 Uninjured fibeip $P$

10 Uninjured fibers

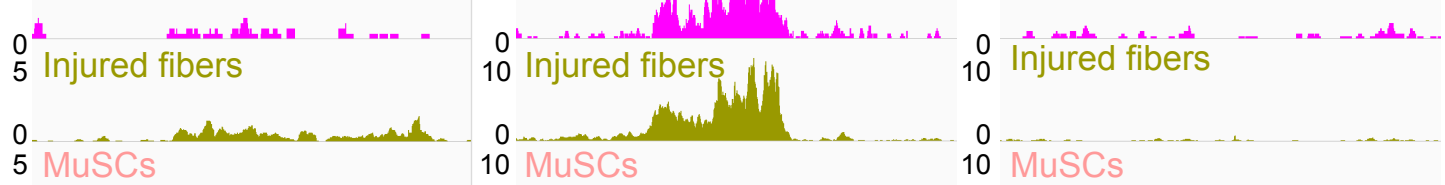

Pou5f1 
Figure 3: Uninjured and injured myofibers and MuSCs display distinct chromatin states

(A)Heatmap clustering of Pearson correlation coefficients showing the correlation between the replicates of the conditions in the regions defined by the union peakset (merged peaks of all replicates/samples). (B) Projection of samples along first two principal components found by PCA showing the separate clustering of different samples and the clustering of each replicate of the same condition together. (C) Pile up analysis of differentially accessible peaks between uninjured myofibers and MuSCs and between injured myofibers and uninjured myofibers. Less accessible regions: FDR $<0.05$ and LFC $<0.5$. More accessible peaks: FDR < 0.05 and LFC >2. (D) Heatmap clustering of Pearson correlation coefficients indicating the correlation between the replicates in the regions defined by the consensus peakset derived from the uninjured myofibers, injured myofibers and MuSCs. (E) Peak annotation pie charts for the differentially accessible peaks between uninjured myofibers vs MuSCs and uninjured myofibers vs injured myofibers. (F) Venn diagram of the number of ATAC-Seq peaks that are unique or overlapping between uninjured myofibers vs MuSCs and uninjured myofibers vs injured myofibers. 
Figure 3

bioRxiv preprint doi: https://doi.org/10.1101/2021.06.14.448380; this version posted June 15, 2021. The copyright holder for this preprint
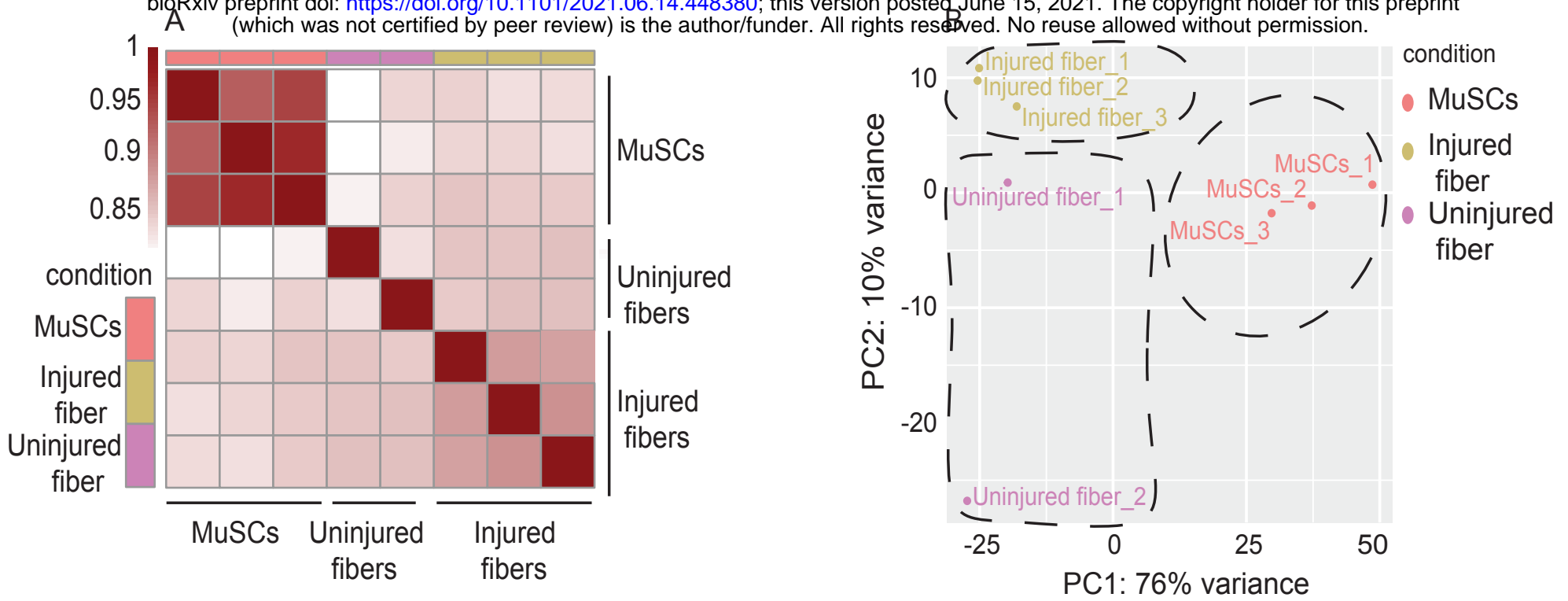

C

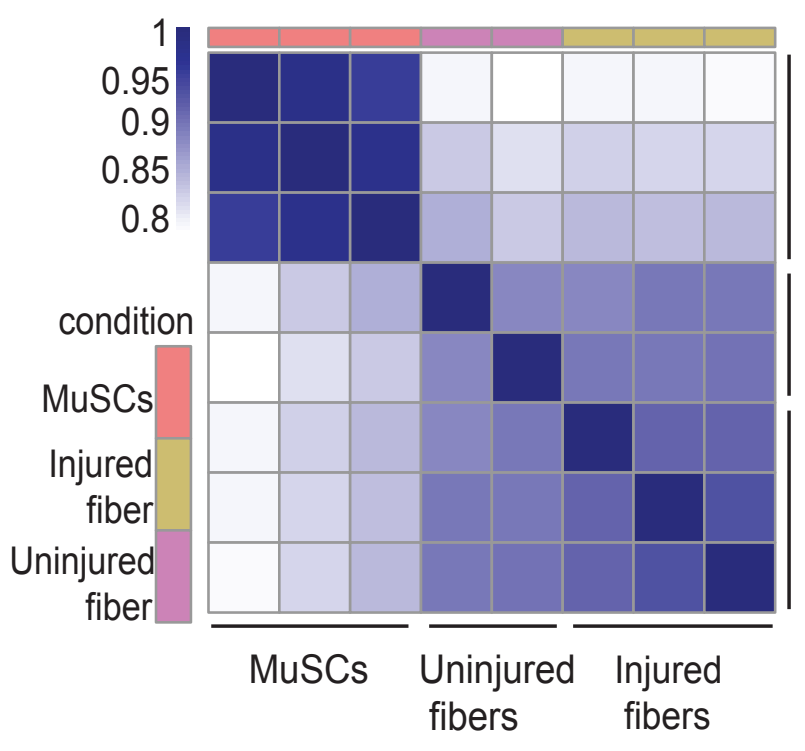

$\mathrm{D}$
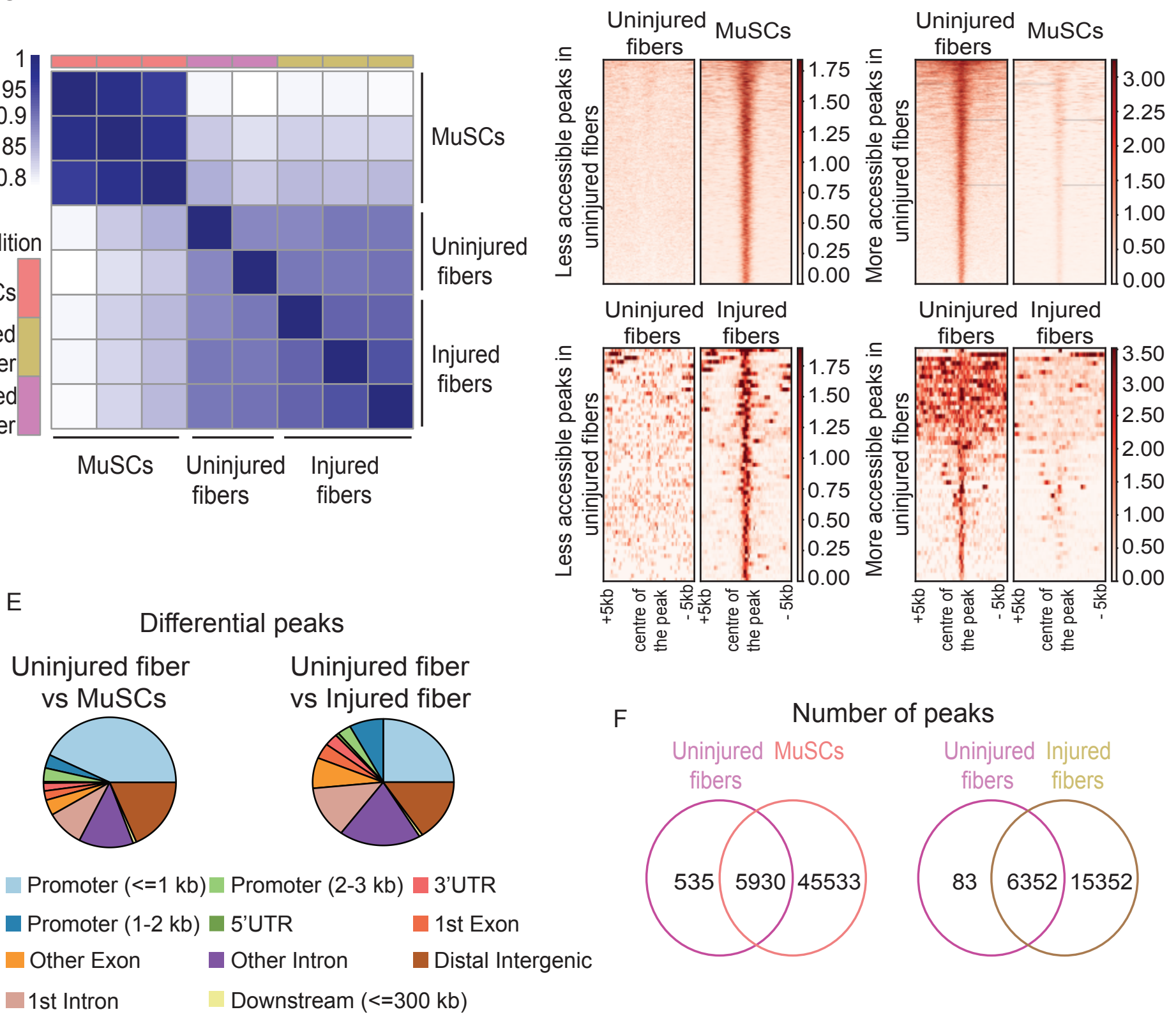

F

Number of peaks

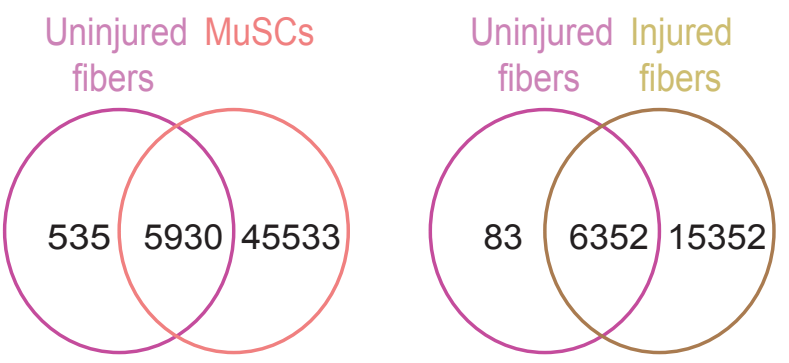


Figure 4: Comparative analysis of chromatin state between uninjured myofibers and MuSCs and between uninjured myofibers and injured myofibers

(A-C) Gene Ontology (GO Biological Process) analysis of genes associated with ATAC-Seq peaks based on association by proximity using Genomic Regions Enrichment of Annotations Tool (GREAT) (84) for all peaks present in the uninjured myofibers, MuSCs and injured myofibers respectively. (D) Volcano plot of differentially accessible regions/peaks identified by FDR $<0.05$ and LFC $>=1$ between uninjured myofibers and MuSCs. Each dot represents a differentially accessible region/peak and the distance to the nearest gene is annotated. (E) Volcano plot of differentially accessible regions/peaks identified by FDR < 0.05 and LFC >= 1 between uninjured myofibers and injured myofibers. Each coloured dot represents a differentially accessible region/peak and the distance to the nearest gene is annotated. 
bioRxiv preprint doi: https://doi.org/10.1101/2021.06.14.448380; th/Fiverige psted June 15, 2021. The copyright holder for this preprint (which was not certified by peer review) is the author/funder.All rights reserved. No reuse allowed without permission.

A

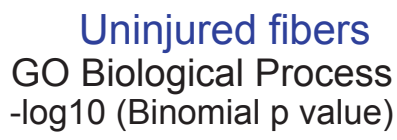

$\begin{array}{llll}0 & 20 & 40 & 60\end{array}$

Striated muscle cell development Muscle cell development Actomyosin structure organization Component assembly in morphogenesis Cardiac muscle cell development Cardiac cell development Regulation of metabolites and energy Polll transcription in response to stress

$\mathrm{mRNA}$ catabolic process
Transcription in response to stress

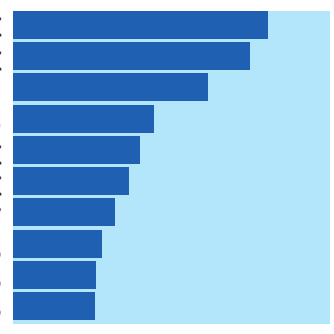

B

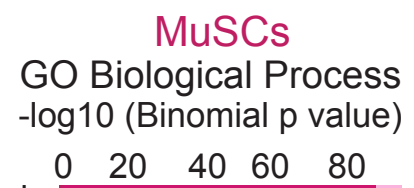

Response to incorrect protein

$\begin{array}{lllll}0 & 20 & 40 & 60 & 80\end{array}$

Mitochondrial membrane permeability
ER unfolded protein response

Cellular response to unfolded protein Adherens junction organization

Regulation of membrane permeability

Regulation of TLR signalling pathway

Mitochondrial transmembrane transport

Execution phase of apoptosis

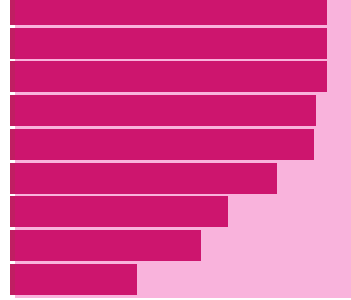

C

Injured fibers

GO Biological Process $-\log 10$ (Binomial $p$ value)

$\begin{array}{llll}0 & 40 & 80 & 120\end{array}$

Striated muscle cell development Myofibril assembly

Sarcomere organization
Muscle cell development

Actomyosin structure organization

Component assembly in morphogenesis Mitochondrial transport

Muscle fiber development

Regulation of metabolites and energy

Cardiac muscle cell development
D

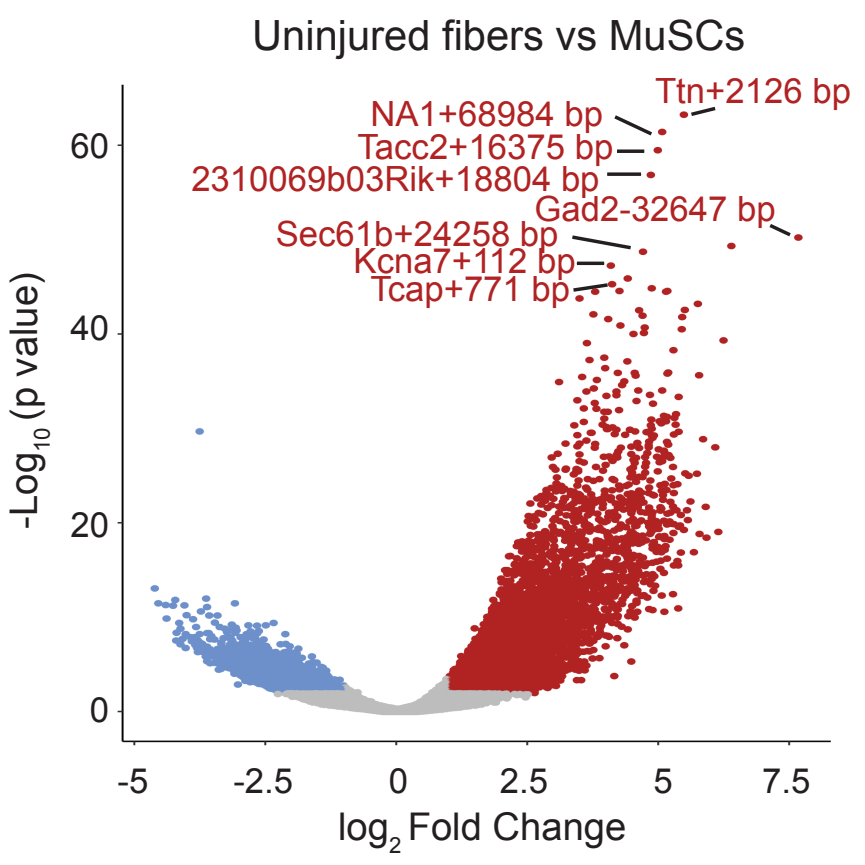

E

Injured fibers vs Uninjured fibers

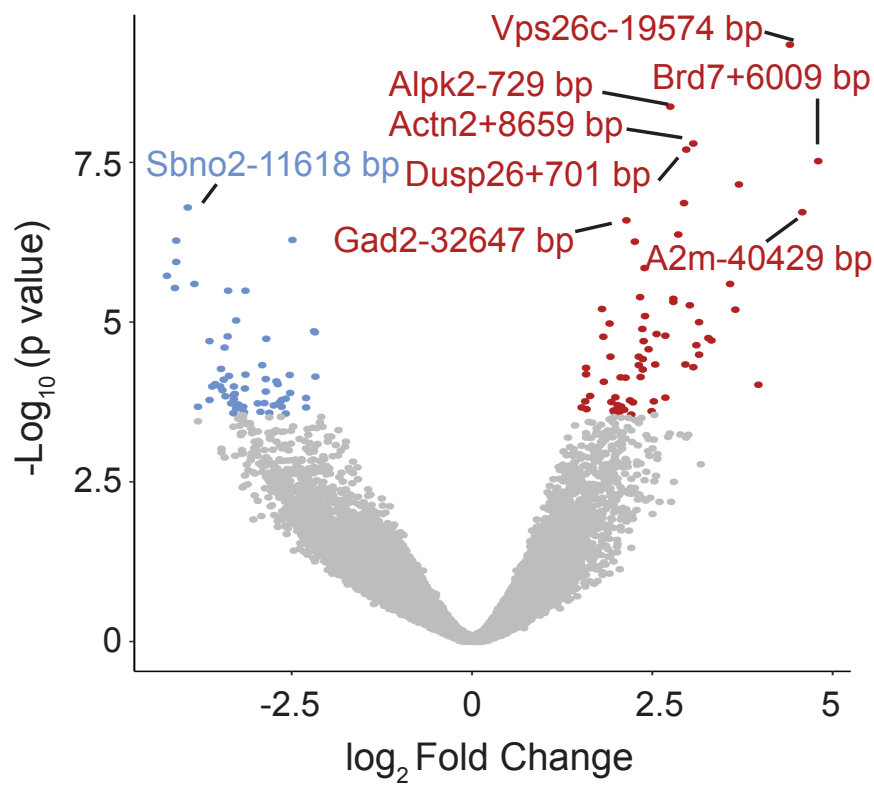


Figure 5: Identification of cell type specific pathways by global analysis of chromatin accessibility

(A) Gene Set Enrichment Analysis performed on nearby genes of differentially accessible regions/peaks for uninjured myofibers compared to injured myofibers. Top 10 enriched pathways are shown although do not reach significance. (B) Gene Set Enrichment Analysis performed on nearby genes of differentially accessible regions/peaks for uninjured fibers compared to MuSCs. Top 10 significantly enriched pathways are shown (FDR < 0.01). (C) Heatmap for myogenesis based on read counts of MuSCs and uninjured fibers $+/-1 \mathrm{~kb}$ of the TSS of each gene in the myogenic pathway. (D) IGV snapshot of Tropomyosin 2 (Tpm2). (E) IGV snapshot of Glutathione Peroxidase 3 (Gpx3). 
bioRxiv preprint doi: https://doi.org/10.1101/2021.06.14.448380; thisudurers 5 5 posted June 15, 2021. The copyright holder for this preprint (which was not cAtified by peer review) is the author/funder. All rights reserved. No reuse allowed withut permission.

\section{Uninjured fibers vs Injured fibers}

Fatty acid metabolism Oxidative phosphorylation

EMT

IFNy response Myogenesis

Complement Early estrogen response

Coagulation Inflammatory response IL2-Stat5 signalling

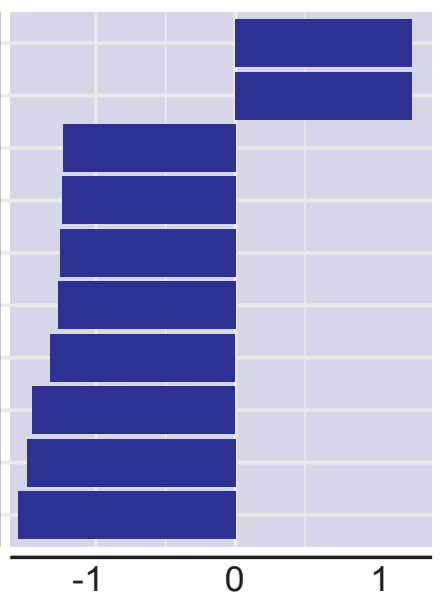

Normalized Enrichment Score C
Uninjured fibers vs MuSCs

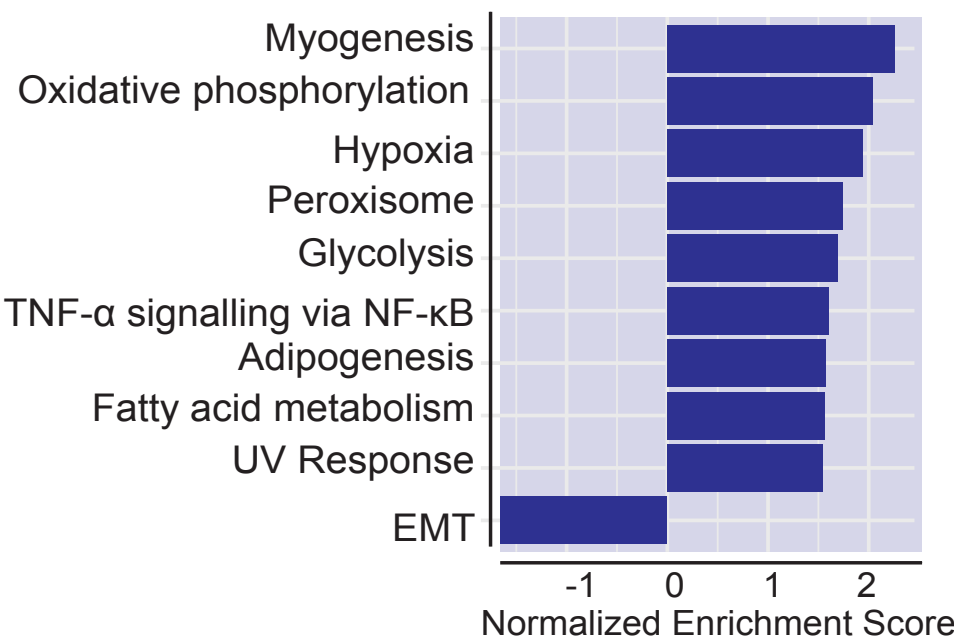

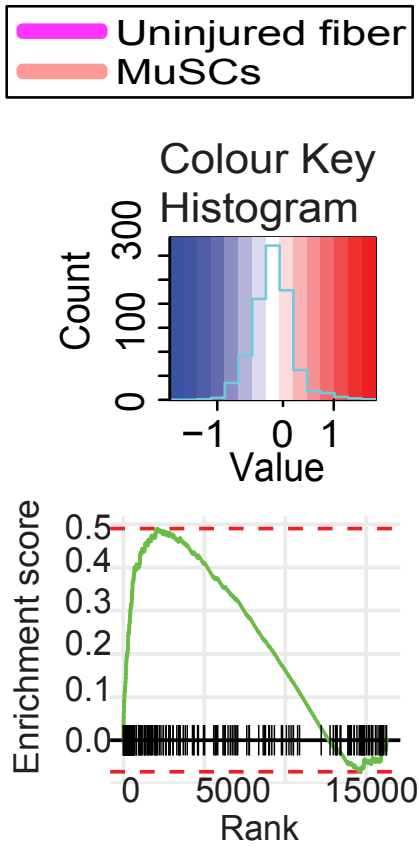

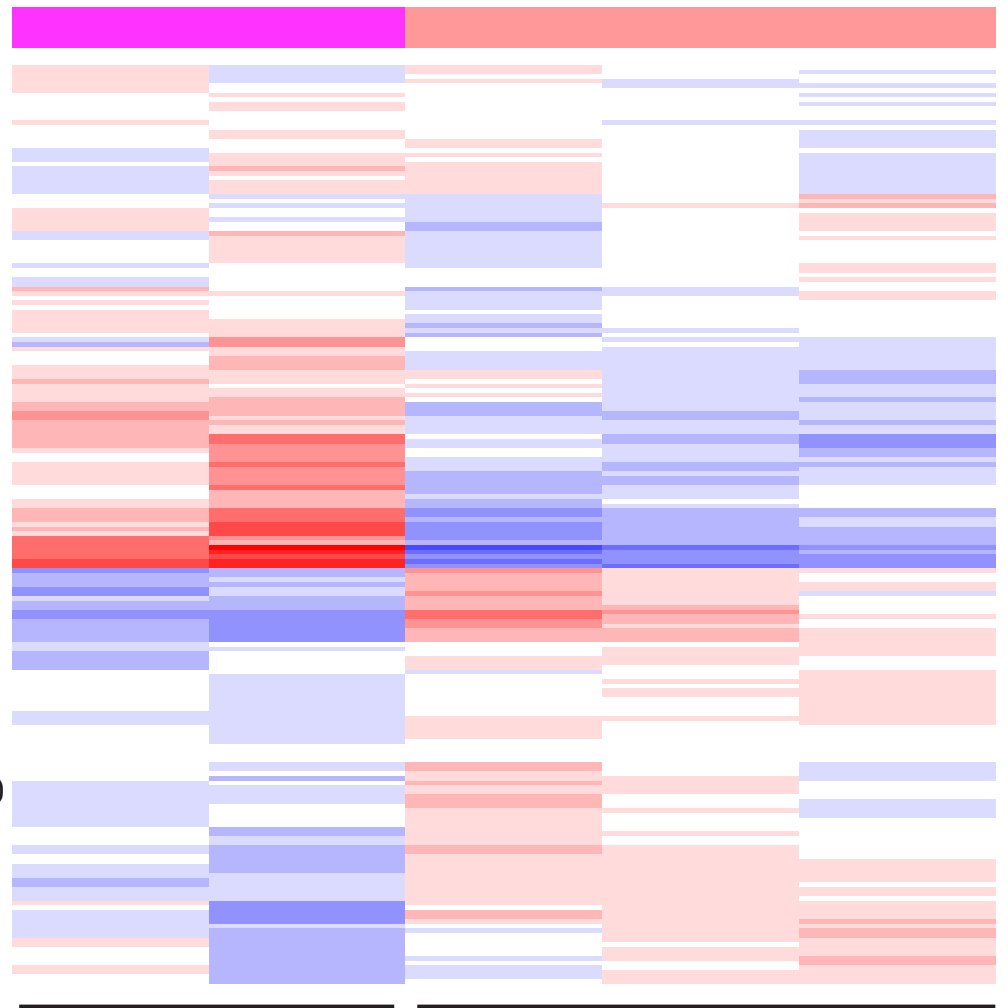

Uninjured fibers

D

15

Uninjured fibers
MuSCs

\section{E}

8 Uninjured fibers

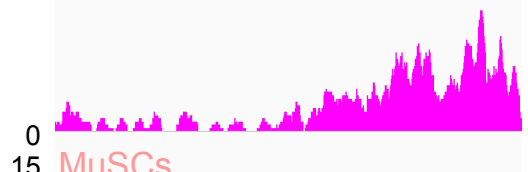

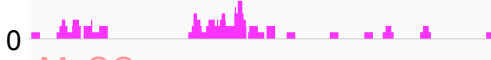
8 MuSCs

0
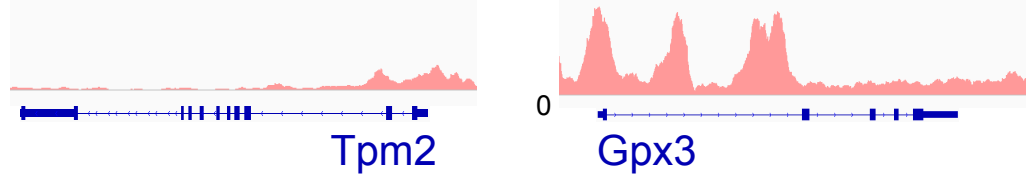


\section{Figure S1: Quality control of ATAC-Seq libraries}

(A) Bioanalyzer profile of an ATAC-Seq library prepared from 5000 MuSCs. (B) Example bioanalyzer profile of ATAC-Seq library prepared from a single myofiber. (C) Representative picture of a ready to sequence MuSC ATAC-seq library after size selection, visualized on an agarose gel. (D) Fold enrichment for the TSS of MyoD compared to negative control region of Chromosome 17 qE5 between MuSC ATAC-Seq libraries and untagmented DNA as seen by qPCR $(n=5,3$ biological replicates and 2 technical duplicates), two-tailed t-test, error bars $= \pm \mathrm{SD}$. (E) Representative picture of a ready to sequence ATACseq on a single myofiber library after size selection, visualized on an agarose gel. (F) qPCR for the TSS of MyoD compared with a negative control region of Chromosome 17 qE5 for the ATAC-Seq libraries prepared from single myofibers $(n=3$, biological triplicates), two-tailed t-test, error bars $= \pm S$. 


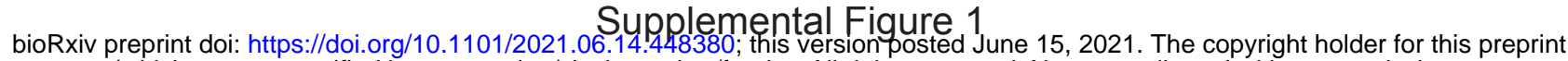
(which was not certified by peer review) is the author/funder. All rights reserved. No reuse allowed without permission.

A

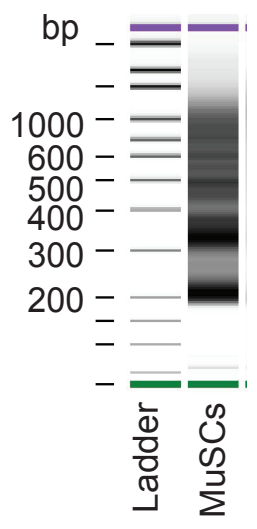

C

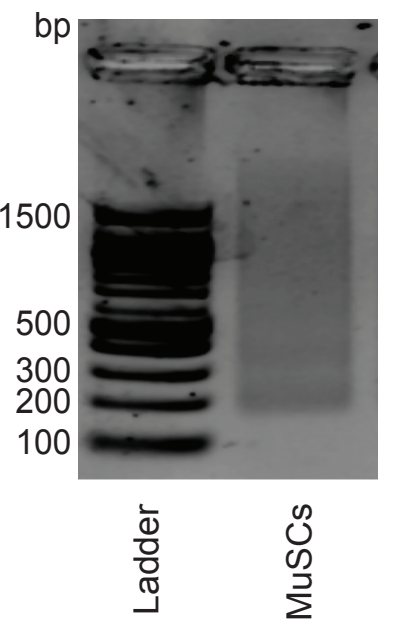

MuSCs

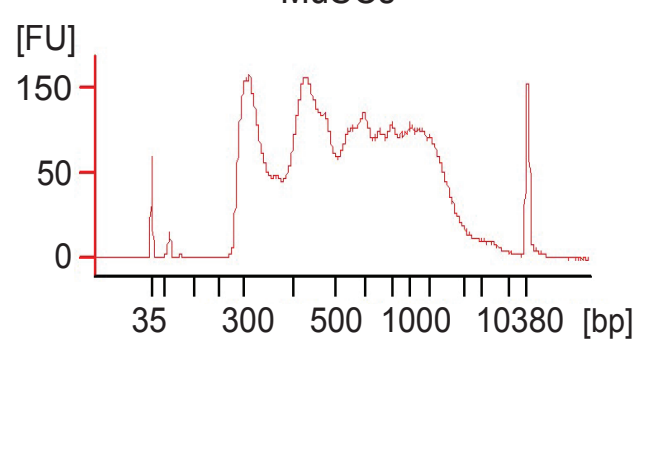

D

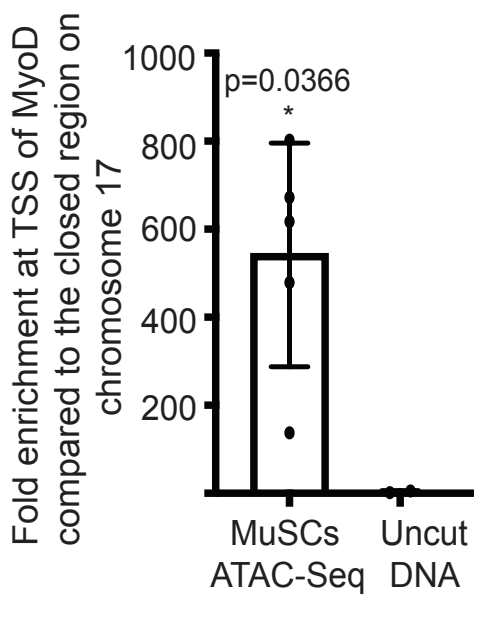

B

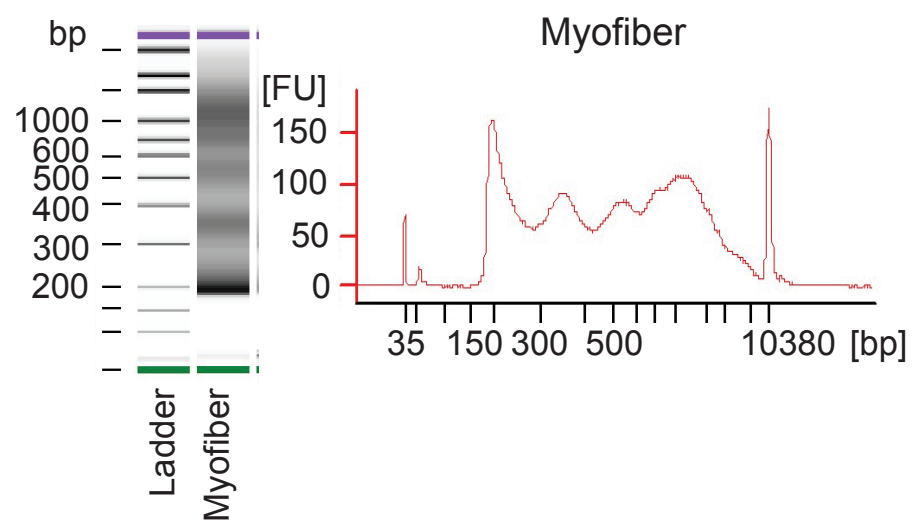

$\mathrm{E}$

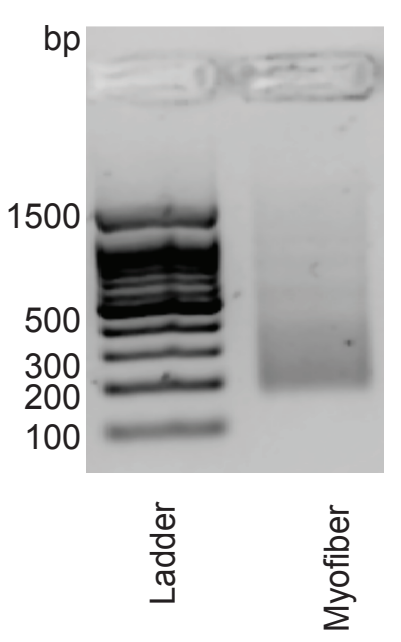

$\mathrm{F}$

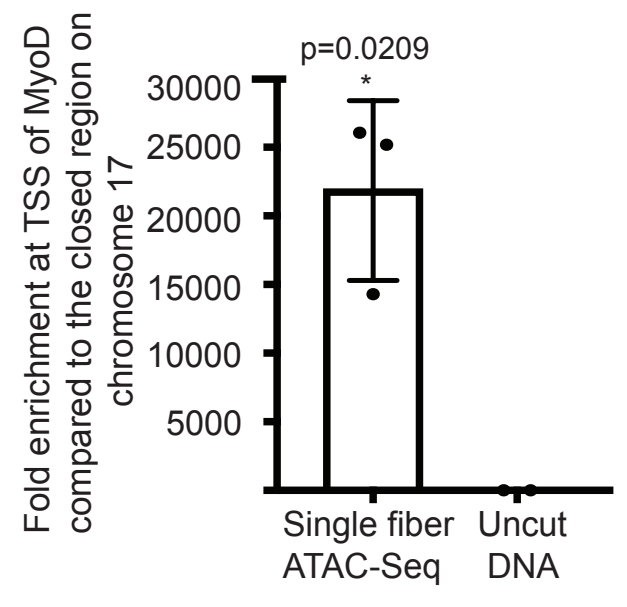




\section{Figure S2. IGV snapshots of non-myogenic genes}

(A) Platelet and Endothelial Cell Adhesion Molecule 1 (Pecam1) expressed in endothelial cells. (B) Resistin (Retn) as a marker of adipocytes. (C) CD45 expressed in hematopoietic cells. (D) CD90 (Thyl) expressed in fibroblasts. (E) Lymphocyte antigen 6a (Ly6a) expressed in fibro/adipogenic progenitors (FAPs). (F) Adhesion G protein-coupled receptor E1 (ADGRE1) gene expressed in macrophages. (G) The housekeeping gene RPS2 used as a positive control. (H) Housekeeping gene TATA-Box Binding protein $(\mathrm{Tbp})$ was also used as a positive control. 


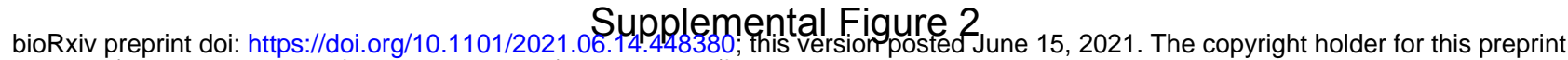
(which was not certified by peer review) is the author/funder. All his reserved. No reuse allowed without permission.
10 Uninjured fibers
10 Uninjured fibers

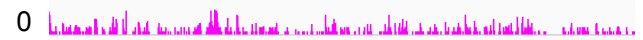

10 Injured fibers

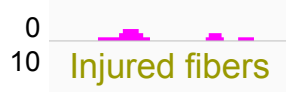

10 MuSCs

${ }_{10}^{0}$ MuSCs

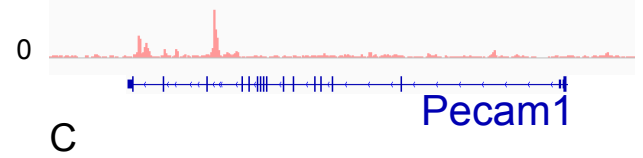

10 Uninjured fibers

0

D

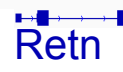

10 Uninjured fibers

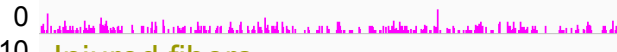

10 Injured fibers

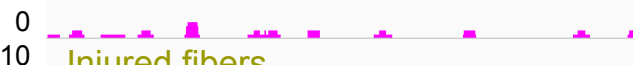

10 Injured fibers

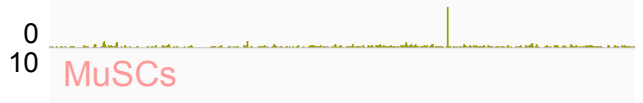

$10-1-2$

0

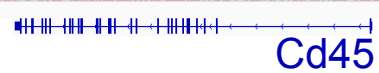

\section{$\mathrm{E}$}

0

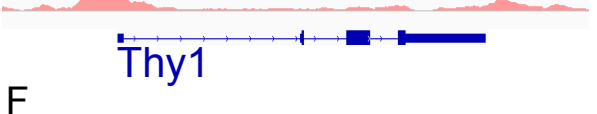

10 Uninjured fibers

10 Uninjured fibers

10 Injured fibers

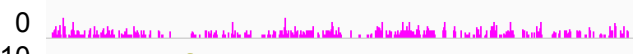

10 Injured fibers

$1 0 \longdiv { \text { MuSCs } }$

10 MuSCs

0

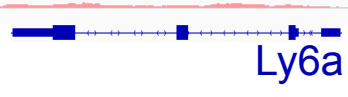

\section{G}

0

$\mathrm{H}$

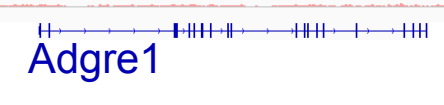

5 Uninjured fibers

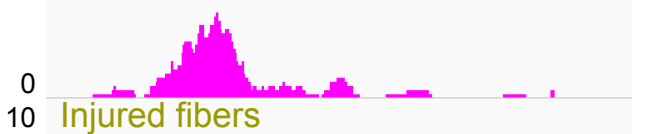

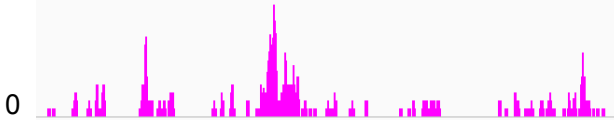
5 Injured fibers
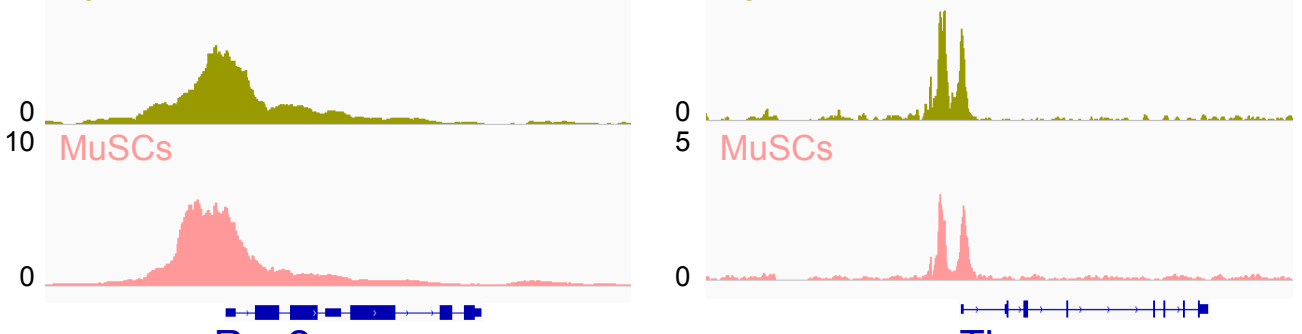

Rps2

Tbp 


\section{Figure S3: Correlation analysis between biological replicates of each condition}

(A-C) IGV snapshots of genes expressed in myofibers and MuSCs for all the replicates of each condition that were pooled together for further analysis. DNase-Seq track added to demonstrate correlation of the myofiber ATAC-Seq with previously performed DNase-Seq on skeletal muscle. (A) Housekeeping gene Gapdh. (B) The muscle creatine kinase (Ckm). (C) Myogenic Factor 5 (Myf5). (D-J) Scatter plot showing the Pearson correlation between the replicates. 


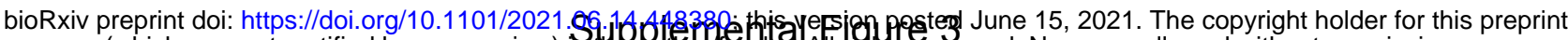
(which was not certified by peer review) is thlequthorkunder. All hights eserved. No reuse allowed without permission.

A

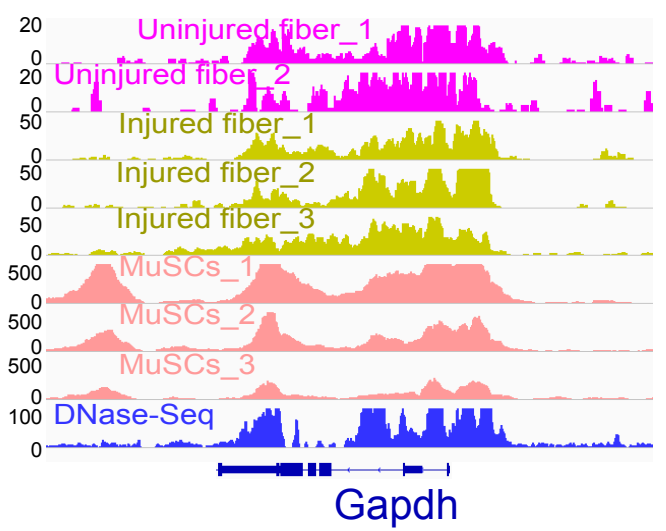

D

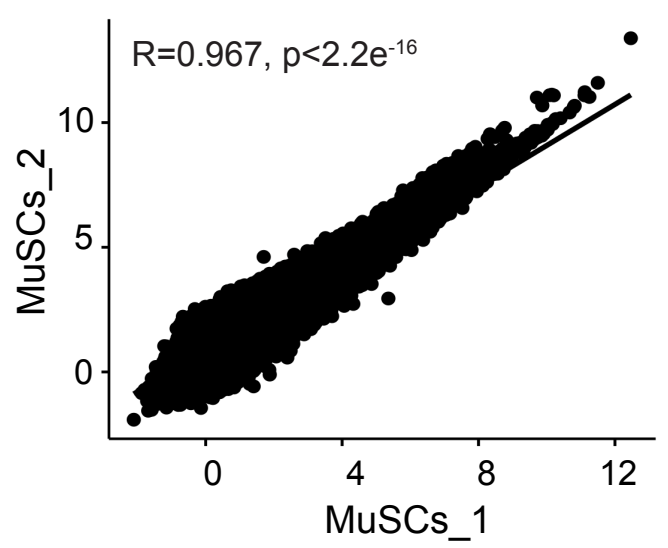

G

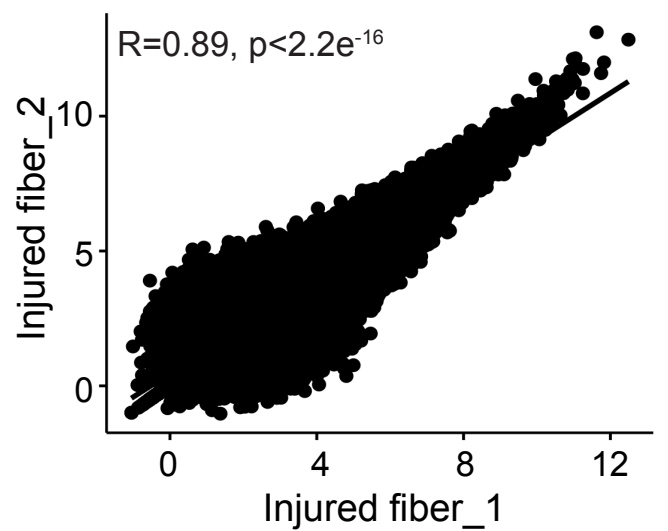

\section{$\mathrm{B}$}

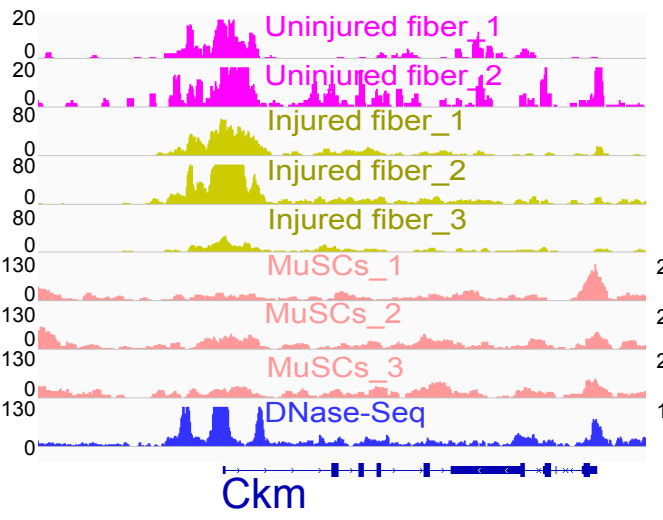

$\mathrm{E}$

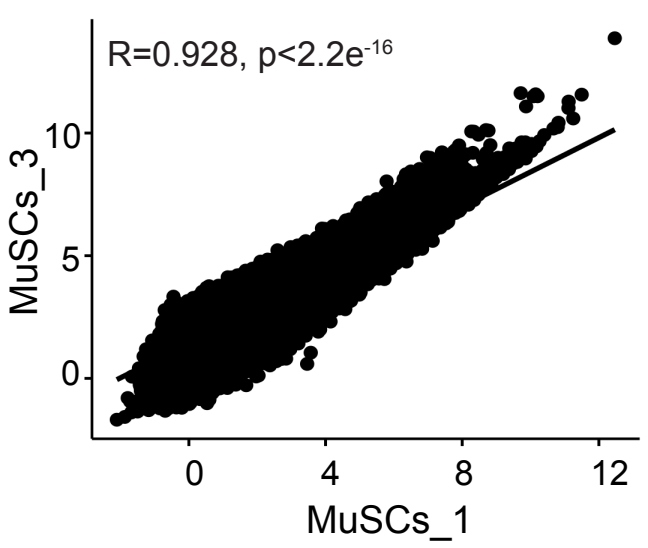

$\mathrm{H}$

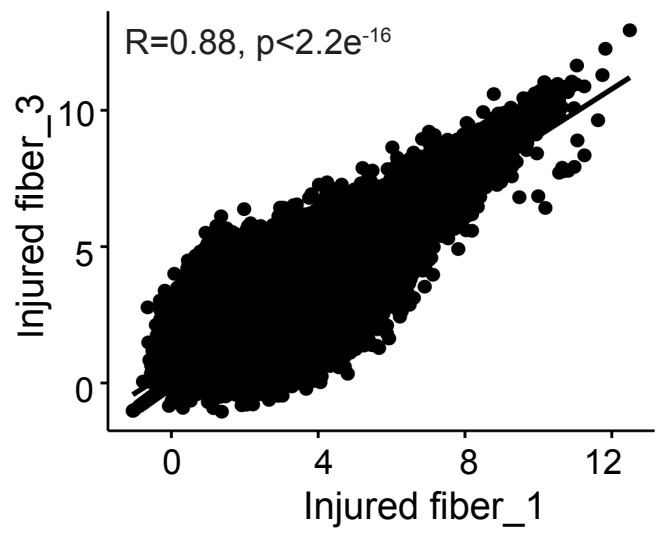

J

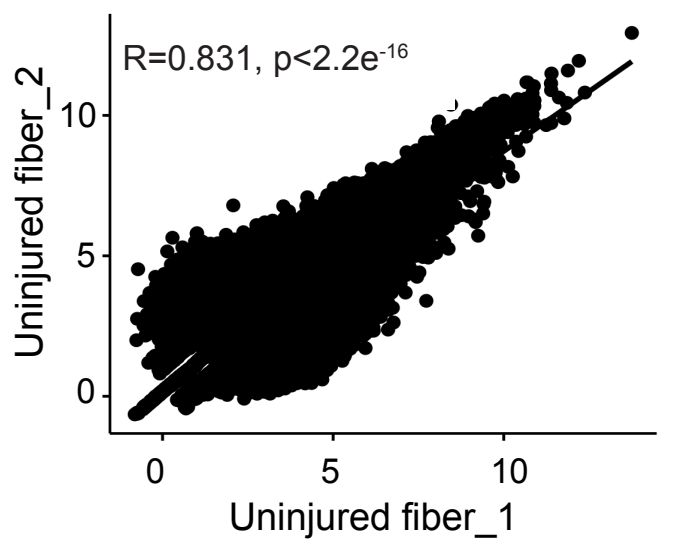

C

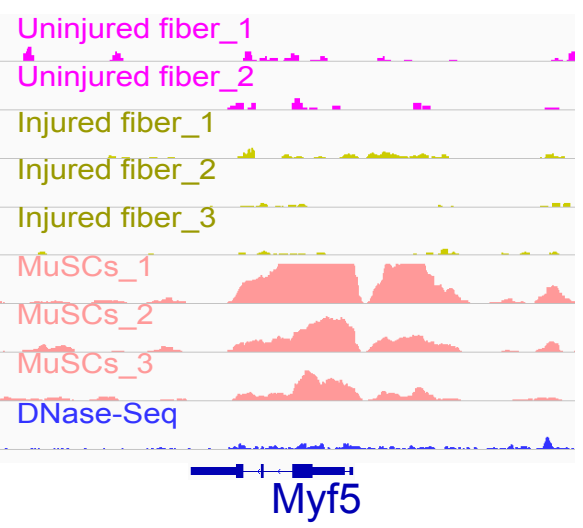

$\mathrm{F}$

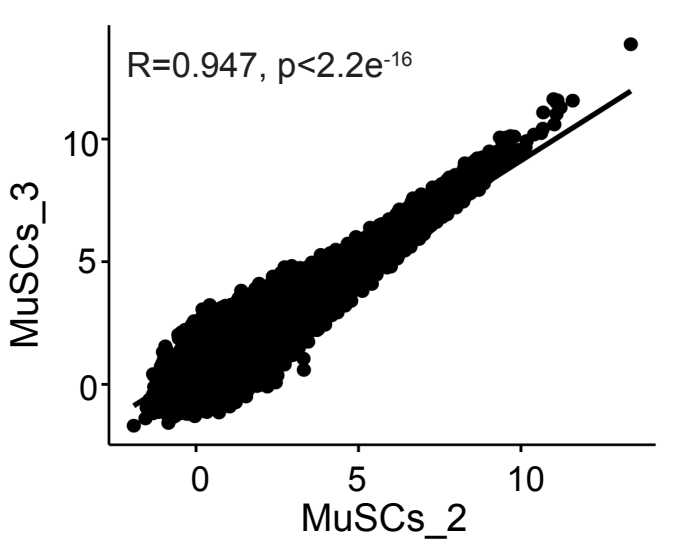

I

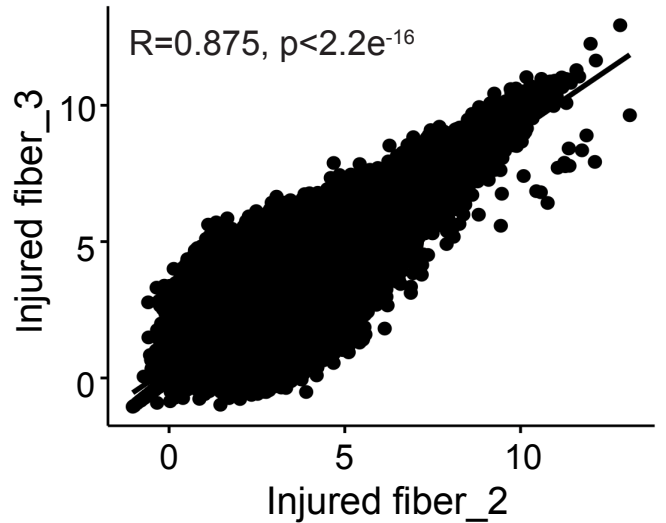


bioRxiv preprint doi: https://doi.org/10.1101/2021.06.14.448380; this version posted June 15, 2021. The copyright holder for this preprint (which was not certified by peer review) is the author/funder. All rights reserved. No reuse allowed without permission.

Figure S4. IGV snapshots of genes expressed in fast and slow muscle fiber types

(A) Troponin 12 (Tnni2) expressed in fast skeletal muscle fiber. (B) Troponin T3 (Tnnt3) expressed in fast skeletal muscle fiber. (C) Troponin T1 (Tnnt1) expressed in slow skeletal muscle fibers. (D) Myosin heavy chain 7 (Myh7) expressed in slow skeletal muscle fibers 


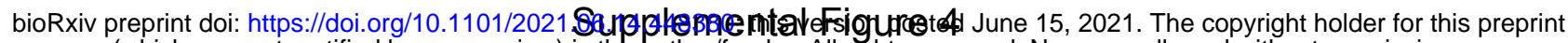
(which was not certified by peer review) is the author/funder. All rights reserved. No reuse allowed without permission.

\section{A}

10

Uninjured fibers

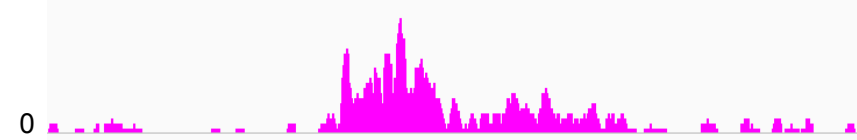

10 Injured fibers

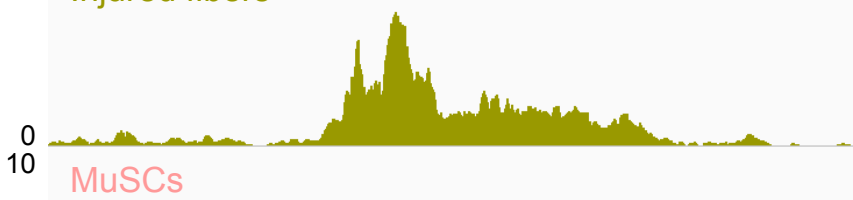

0

\section{Thnil +1+H}

Tnni2

\section{C}

Uninjured fibers

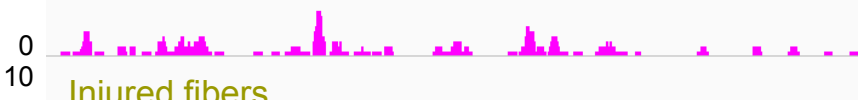
Injured fibers

10

$1 0 \longdiv { \text { MuSCS } }$

0

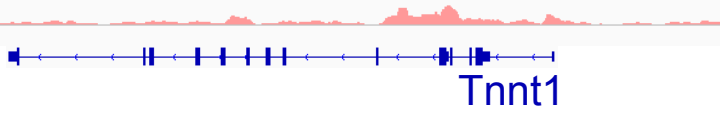

B

Uninjured fibers

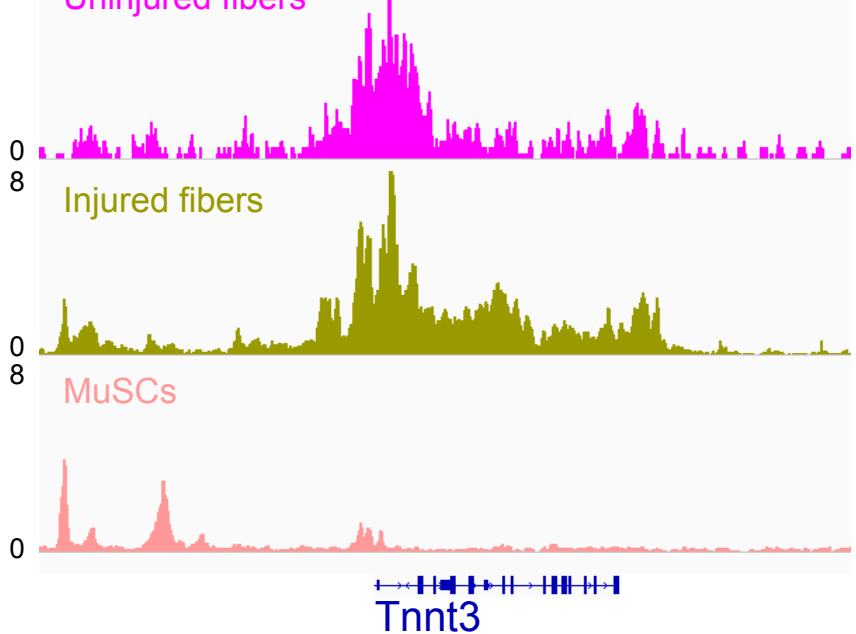

10

Uninjured fibers

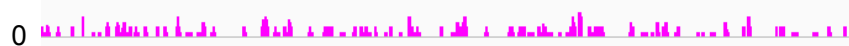
10 Injured fibers

10 MuSCs

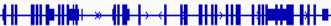

Myh7 
Figure S5: Unique peaks between different conditions indicate a distinct chromatin state for each cell type

(A) Heatmap showing the read count $+/-500 \mathrm{bp}$ of the center of unique peaks to MuSCs compared to uninjured myofibers. (B) Heatmap showing the read count $+/-500 \mathrm{bp}$ of the center of unique peaks to injured myofibers compared to uninjured myofibers. (C) Peak score distribution (calculated by MACS2 peak calling algorithm) for each of the different conditions. Peak score $=-\log 10$ (FDR). 


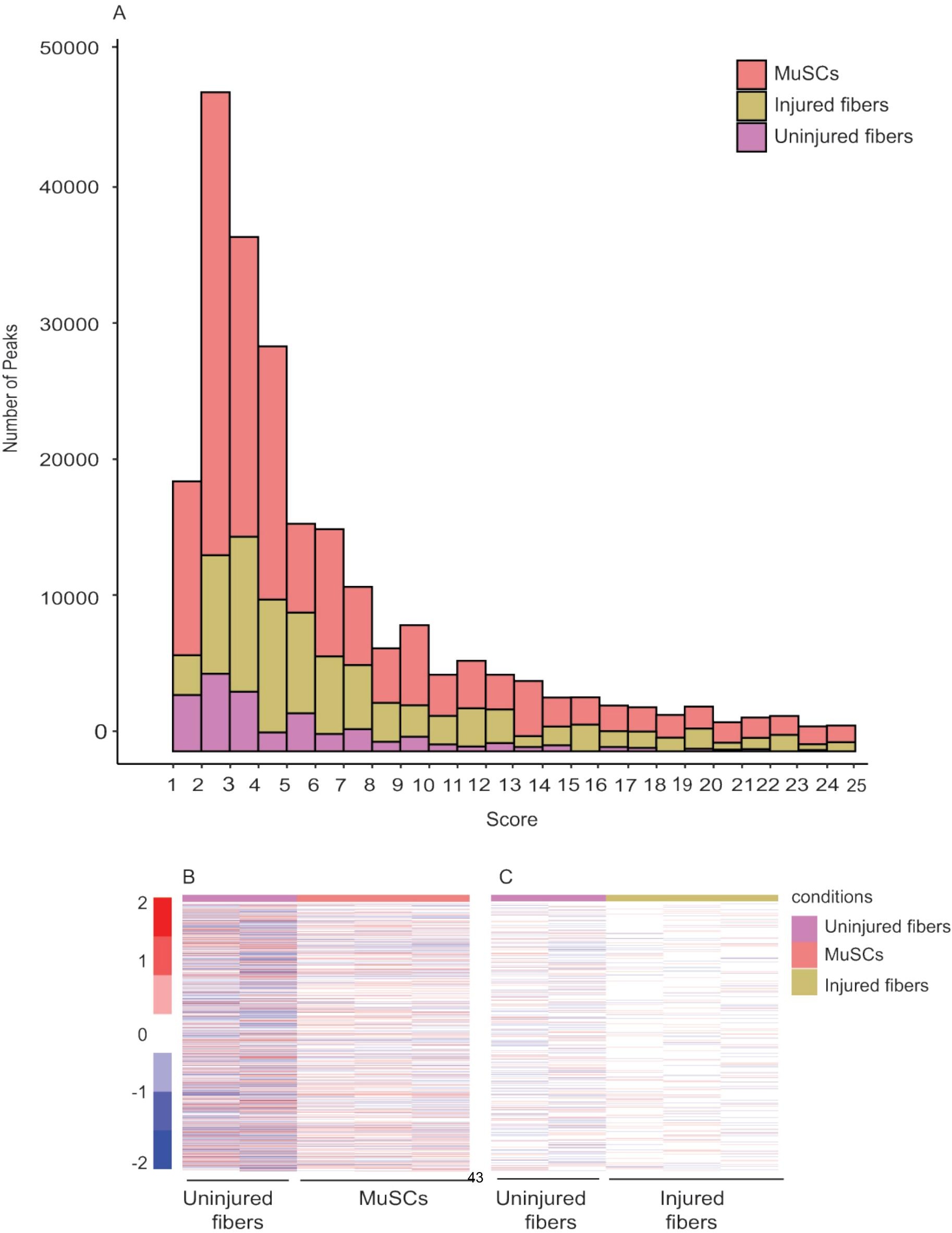




\section{Figure S6: Gene Ontology analysis of unique and common peaks between conditions}

(A) Gene Ontology (GO Biological Process) analysis of genes associated with unique peaks present in the uninjured myofiber compared to MuSCs, based on the proximity of the peaks to the genes. (B) GO term analysis of genes associated with unique peaks in MuSCs compared to uninjured myofibers. (C) GO term analysis of genes associated with peaks that are common between MuSCs and uninjured myofibers. (D) GO term analysis of genes associated with peaks that are common between injured and uninjured myofibers. (E) GO term analysis of genes associated with unique peaks in injured myofibers compared to uninjured myofibers. (F) GO term analysis of genes associated with unique peaks in uninjured myofibers compared to injured myofibers. 


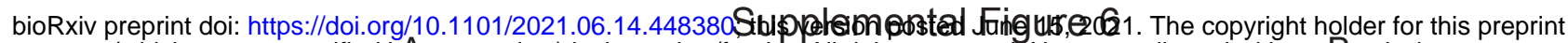
(which was not certified bApeer review) is the author/funder. All rights reserved. No reuse allowed without Bermission.

\section{Peaks unique to uninjured fibers compared to MuSCs \\ GO Biological Process $-\log 10$ (Binomial $p$ value)}

Sarcomere organization Myofibril assembly

Striated muscle cell development

Muscle cell development Actomyosin structure organization Cardiac muscle fiber development Muscle thin filament assembly Striated muscle cell differentiation Assembly in morphogenesis Muscle fiber development

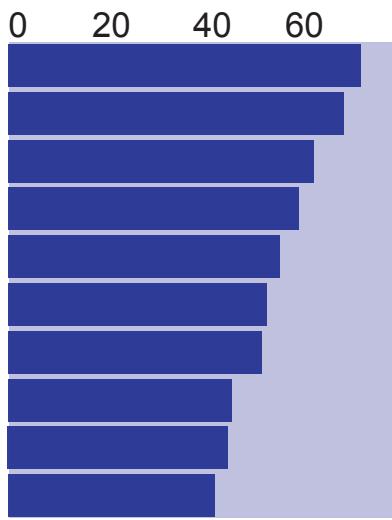

C

Peaks common to MuSCs and uninjured fibers GO Biological Process $-\log 10$ (Binomial $p$ value)

Mitochondrial transport $0 \quad 10-20$

Pol II transcription in stress Pol II transcription in ER stress

Pol II transcription regulation

Response to incorrect protein Cell response to incorrect protein Transcription in response to stress Regulation of metabolites and energy Mitochondrial membrane organization Protein folding

\begin{tabular}{ll}
$0 \quad 10 \quad 20$ \\
\hline \\
\hline \\
\hline \\
\hline \\
\hline \\
\hline \\
\hline
\end{tabular}

Peaks unique to MuSCs compared to uninjured fibers

GO Biological Process - $\log 10$ (Binomial $p$ value)

Adherens junction organization Regulation of myotube differentiation Mitochondrial membrane permeability Regulation of Notch signalling Regulation of membrane permeability Regulation of cytochrome c frelease Regulation of ERBB signalling pathway Regulation of collagen metabolism Unsaturated fatty acid biosynthesis Regulation of collagen biosynthesis

\section{$\begin{array}{lllll}0 & 20 & 40 & 60 & 80\end{array}$}

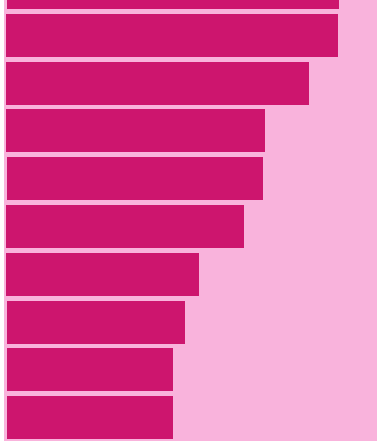

D

Peaks common to injured and uninjured fibers GO Biological Process -log10 (Binomial $p$ value)

Myofibril assembly
Sarcomere organization
Striated muscle cell development
Actomyosin structure organization
Muscle cell development
Mitochondrial transport
Muscle fiber development
Assembly in morphogenesis
Nucleosome assembly
Nucleosome organization

0 $\begin{array}{lll}15 & 30 & 45\end{array}$

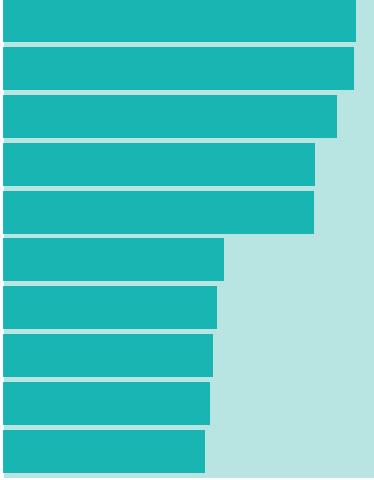

\section{E}

F

Peaks unique to injured fibers compared to uninjured fibers

GO Biological Process $-\log 10$ (Binomial $p$ value)

$$
0
$$

Striated muscle cell development

Myofibril assembly

Muscle cell development

Actomyosin structure organization

Sarcomere organization

Assembly in morphogenesis Regulation of metabolites and energy

Muscle fiber development

Cardiac cell development Cardiac muscle celld development

$\begin{array}{llll}0 & 40 & 80 & 110\end{array}$

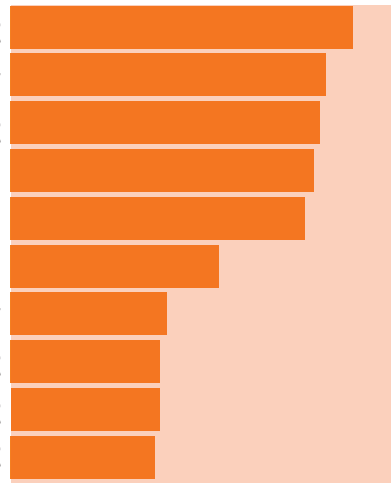

Peaks unique to uninjured fibers compared to injured fibers

GO Biological Process $-\log 10$ (Binomial $p$ value)

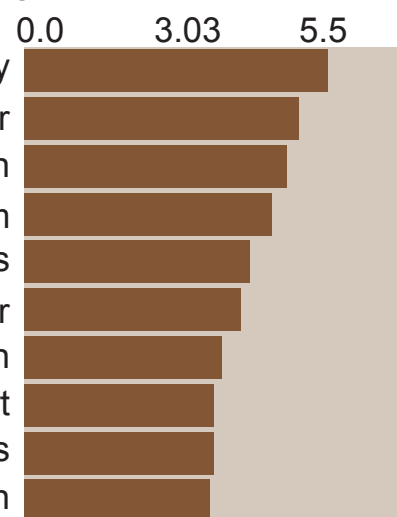


Figure S7: Analysis of Notch and TGF $\beta$ signalling pathways reveal differential accessibility between MuSCS and uninjured myofibers and injured and uninjured myofibers

(A)Heatmap showing genes involved in the Notch signalling pathway based on read counts of MuSCs, uninjured fibers and injured fibers, +/- $1 \mathrm{~kb}$ of the TSS of each gene in the pathway. (B) IGV snapshot of Notch homolog 1 (Notch1). (C) IGV snapshot of Protein jagged 2 (Jag2). (D) Heatmap showing genes involved in the TGF $\beta$ signalling pathway based on read counts of MuSCs, uninjured fibers and injured fibers, +/- $1 \mathrm{~kb}$ of the TSS of each gene in the pathway. (E) IGV snapshot of Noggin (Nog). (F) IGV snapshot of Bone morphogenetic protein (Bmp4). 


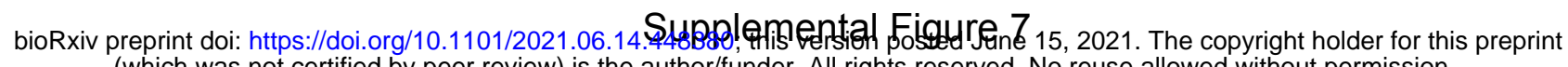
(which was not certified by peer review) is the author/funder. All rights reserved. No reuse allowed without permission.

A
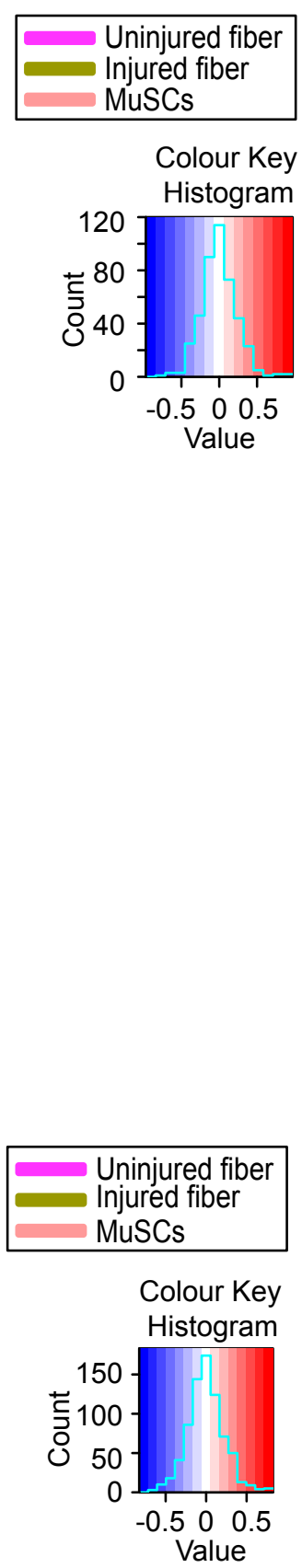

$\mathrm{D}$
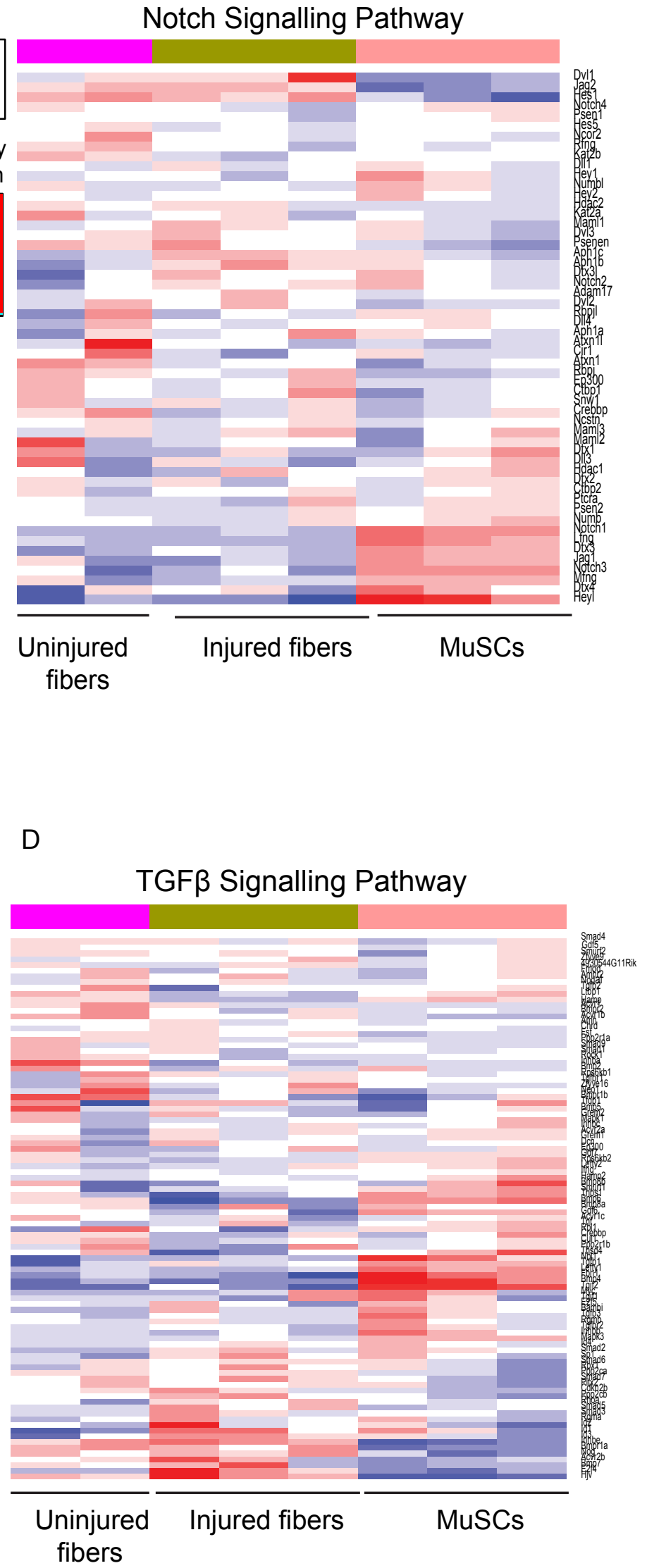

B

7 Uninjured fibers

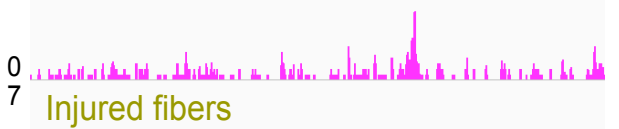
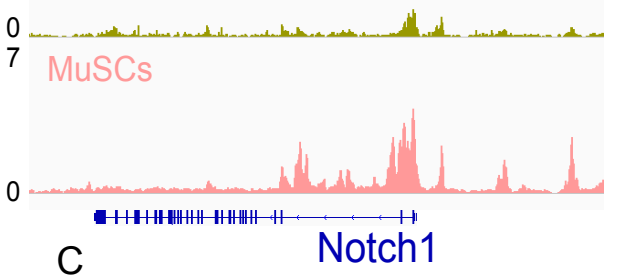

8 Uninjured fibers

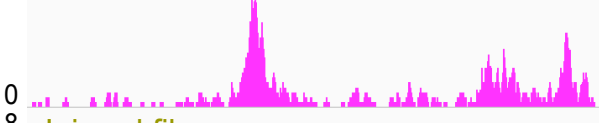

8 Injured fibers
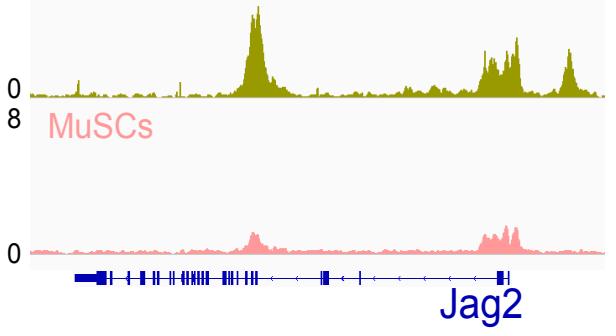

$E$

6 Uninjured fibers

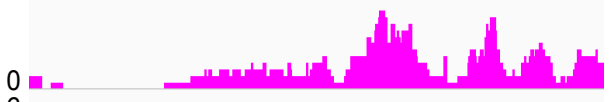

6 Injured fibers
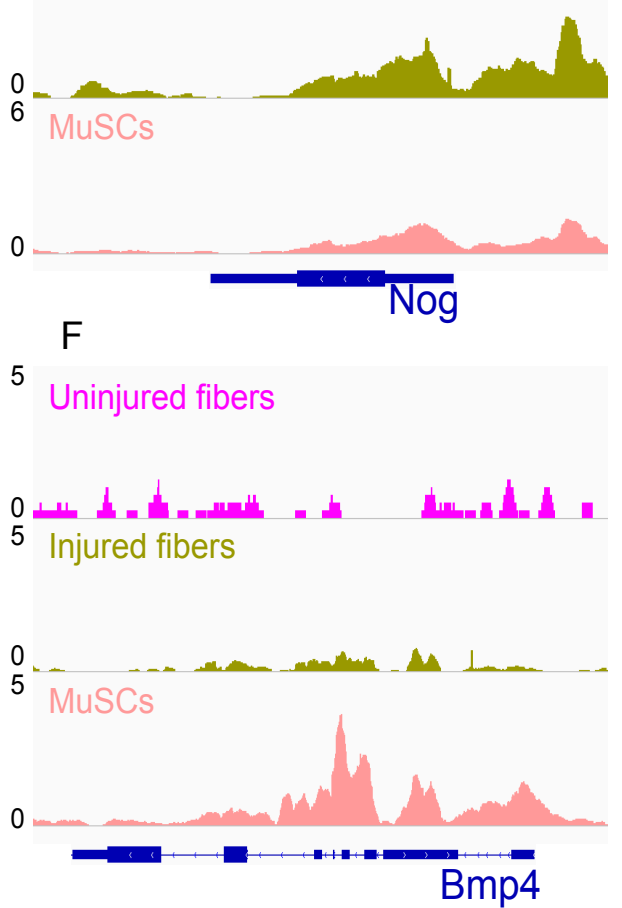
Table 1: Sequencing read information for smfATAC-Seq and MuSCs ATAC-Seq libraries

\begin{tabular}{|c|c|c|c|c|c|c|c|c|c|}
\hline Library & $\begin{array}{l}\text { Number of } \\
\text { Raw Reads }\end{array}$ & $\begin{array}{l}\text { Number of } \\
\text { Surviving Reads }\end{array}$ & $\begin{array}{l}\text { Aligned Filtered } \\
\text { Reads (mm10 } \\
\text { reference) }\end{array}$ & $\begin{array}{l}\text { Duplicate } \\
\text { Reads }\end{array}$ & $\begin{array}{l}\text { Mitochondrial } \\
\text { Reads }\end{array}$ & $\begin{array}{l}\text { Percentage of } \\
\text { Mitochondrial } \\
\text { Reads (\%) }\end{array}$ & $\begin{array}{l}\text { Final Reads } \\
\text { Aligned }\end{array}$ & $\begin{array}{l}\text { Number } \\
\text { of Peaks }\end{array}$ & $\begin{array}{l}\text { Fraction in } \\
\text { peaks (FrIP) }\end{array}$ \\
\hline \multicolumn{10}{|l|}{ Muscle Stem } \\
\hline Cells_1 & $1.76 \mathrm{E}+08$ & $1.14 \mathrm{E}+08$ & 103130186 & 47623836 & 529967 & 0.51 & $5 E+07$ & 65568 & 0.364 \\
\hline \multicolumn{10}{|l|}{ Muscle Stem } \\
\hline Cells_2 & $1.75 \mathrm{E}+08$ & $1.17 \mathrm{E}+08$ & 103570009 & 43672484 & 374176 & 0.36 & $6 \mathrm{E}+07$ & 68658 & 0.197 \\
\hline \multicolumn{10}{|l|}{ Muscle Stem } \\
\hline Cells_3 & $1.32 \mathrm{E}+08$ & 91261584 & 79944121 & 31299456 & 223540 & 0.28 & $5 E+07$ & 69573 & 0.13 \\
\hline Injured_1 & $2.3 E+08$ & $1.17 \mathrm{E}+08$ & 90040002 & 81024926 & 830215 & 0.92 & $8 \mathrm{E}+06$ & 32853 & 0.289 \\
\hline Injured_2 & $1.95 E+08$ & $1.3 \mathrm{E}+08$ & 98752157 & 88549329 & 1300615 & 1.32 & $9 \mathrm{E}+06$ & 28351 & 0.286 \\
\hline Injured_3 & $1.42 \mathrm{E}+08$ & 62888552 & 52132455 & 42271079 & 868808 & 1.67 & $9 E+06$ & 25002 & 0.233 \\
\hline Uninjured_1 & $1.45 E+08$ & 75781456 & 61034569 & 52588315 & 1274332 & 2.09 & $7 E+06$ & 12276 & 0.218 \\
\hline Uninjured_2 & $1.51 E+08$ & 64192706 & 50120282 & 45914841 & 965037 & 1.93 & $3 E+06$ & 14742 & 0.321 \\
\hline
\end{tabular}


Table 2: Percentage of total peaks found in each genomic feature

\begin{tabular}{lrrr} 
& & \multicolumn{2}{c}{ Uninjured Myofiber } \\
& Muscle Stem Cells (\%) & Injured Myofiber (\%) & $(\%)$ \\
Promoter $(<=1 \mathrm{~kb})$ & 20.66 & 31.61 & 56.54 \\
Promoter (1-2 kb) & 4.81 & 4.84 & 3.45 \\
Promoter (2-3 kb) & 4.37 & 3.92 & 3.01 \\
5'UTR & 0.34 & 0.27 & 0.23 \\
3'UTR & 2.50 & 1.82 & 1.15 \\
1 st Exon & 1.83 & 1.47 & 1.53 \\
Other Exon & 4.75 & 3.42 & 2.19 \\
1 st Intron & 11.85 & 10.87 & 7.35 \\
Other Intron & 20.80 & 18.84 & 10.35 \\
Downstream $(<=300$ & & & \\
kb) & 1.16 & 1.01 & 0.69 \\
Distal Intergenic & 26.95 & 21.93 & 13.51
\end{tabular}


Table 3: Percentage of differential peaks found in each genomic feature

Uninjured Myofiber vs. MuSCs

Promoter $(<=1 \mathrm{~kb})$

Promoter (1-2 kb)

Promoter (2-3 kb)

5'UTR

3'UTR

1 st Exon

Other Exon

1 st Intron

Other Intron

Downstream (<=300

$\mathrm{kb})$

Distal Intergenic
Uninjured vs Injured Myofiber

43.07

3.39

3.12

0.37

0.78

1.95

3.12

2.29

3.91

3.85

7.81

9.16

13.28

13.34

18.75

0.83

0.78

18.39 